\title{
A Generalised Landau-Lifshitz Fluctuating Hydrodynamics model for concurrent simulations of liquids at atomistic and continuum resolution
}

\author{
I.A. Korotkin and S.A. Karabasov
}

School of Engineering and Materials Science, Queen Mary University of London

\begin{abstract}
A new implementation of the hybrid molecular dynamics - hydrodynamics methods based on the analogy with two-phase flows is developed that takes into account the feedback of molecular dynamics on hydrodynamics consistently. The consistency is achieved by deriving a discrete system of fluctuating hydrodynamic equations which solution converges to the locally averaged molecular dynamics field exactly in terms of the locally averaged fields. The new equations can be viewed as a generalisation of the classical continuum Landau-Lifshitz Fluctuating Hydrodynamics model in statistical mechanics to include a smooth transition from large-scale continuum hydrodynamics that obeys a Gaussian statistics to all-atom molecular dynamics. Similar to the classical Landau-Lifshitz Fluctuating Hydrodynamics model, the suggested Generalised Landau-Lifshitz Fluctuating Hydrodynamics equations are too complex for analytical solution, hence, a computational scheme for solving these equations is suggested. The scheme is implemented in a popular open-source molecular dynamics code GROMACS and numerical examples are provided for liquid argon simulations in equilibrium conditions and under macroscopic flow effects.
\end{abstract}

Keywords: molecular dynamics, multiscale modelling, hybrid molecular dynamics - continuum mechanics, two-phase flow analogy 


\section{Introduction}

Many fluid mechanics problems comprise multiple scales in space and time with applications ranging from stellar dust dynamics in astrophysics and cloud formation in atmospheric sciences to high-speed jet flows in aircraft engine exhausts and flows in micro and nanofluidic devices. ${ }^{1}$ For solving macroscopic flow problems, continuum fluid mechanics provides a well-established set of computational methods that are commonly branded as Computational Fluid Dynamics (CFD). For example, for large space and time scales relevant for Navier-Stokes equations, using constitutive approximations assumed at the microscopic level, relevant fluid dynamics properties can be approximated by macroscopic Lagrangian parcels of fluids using Smooth Particle Hydrodynamics $(\mathrm{SPH})^{2,3}$ and Lattice Boltzmann Method (LBM) $)^{4,5}$ as well as represented on the Eulerian grid using finite-difference (FD), finite-volume (FV), and finite-element (FE) methods. ${ }^{6-9}$ However, the continuum approximations break down when the smallest characteristic size of the problem approaches the microscopic scale. ${ }^{10}$ On the other hand, there are methods of computational mechanics of discontinua such as Direct Simulation Monte Carlo (DSMC) ${ }^{11}$ for solving rarefied gas flow problems and all-atom Molecular Dynamics (MD) ${ }^{12}$ for dense fluid flows which are suitable for simulating the flow properties at the microscale. However, the cost of these simulations quickly grows with the system size so that such simulations are typically limited to microscopic-size systems. ${ }^{13}$

If the fidelity of simulations is sacrificed for the sake of the computational speed, there are various methods of coarse-graining, upscaling, or averaging which exist. For example, instead of all-atom MD simulations another option is to agglomerate several atoms as one effective particle governed by some effective force potential at the mesoscopic level. At the next fidelity level, the effective particles can be further averaged into macroscopic particles and the unresolved thermal fluctuations interactions are modelled statistically using Dissipative Particle Dynamics and Fluctuating Hydrodynamics 
models ${ }^{14,15}$, which mesoscale models can be viewed as an intermediate step between the Computational Fluid Dynamics and the discrete particle models.

Compared to the coarse-grain methods, which operate with a single scale, there is a distinct family of multiscale methods which operate at variable resolution. Such methods attempt to provide an all-atom resolution of the process of interest in a certain (small) part of the computational domain while concurrently treating the rest of the domain based on some continuum hydrodynamics approximation. In comparison with "single-scale" simulations, the multiscale simulations are very challenging because they require a coupling of models consisting of very different numbers of degrees of freedom. On the other hand, once a suitable coupling is achieved, the multiscale models can not only accelerate the modelling without a notable loss of the model fidelity ${ }^{16}$ but also serve to reduce the unnecessary data complexity in comparison with all-atom molecular dynamics simulations. There are many examples of computationally effective multiscale models which are based on the sequential approach. In this approach, the microscale part of the model is only loosely coupled with continuum fluid dynamics and run ahead of time to provide constitutive relations required for the macroscopic simulation, e.g. in the form of "look-up" tables. ${ }^{17,18}$

However, this approach requires a significant scale separation between the microscopic and the macroscopic parts of the model for its validity. In comparison with the sequential models, the so-called concurrent models, where the interaction between all parts of the multiscale model is treated concurrently, are very challenging to develop to the state when they reach the same computationally efficiency. On the other hand, the concurrent models are applicable to a more general class of problems since they have a less strict requirement on the scale separation in comparison with the sequential models. This explains the continuing efforts for development of efficient concurrent multiscale models in the literature.

Since the 1990s, various attempts to couple the molecular dynamics and continuum hydrodynamics $(\mathrm{CH})$ started to appear in the multiscale literature. ${ }^{19,20}$ In many approaches, the MD model is connected 
to continuum models by a continuum-atomistic overlap region which acts as a boundary separating the two sub-domains of different resolution. In the state variables schemes ${ }^{19,21-23}$, the MD and $\mathrm{CH}$ regions are connected through a finite zone that keeps the conservation of bulk mass and momentum fluxes. In the flux coupling schemes ${ }^{15}$, a control interface is used to exchange the conservation fluxes between the hydrodynamic and atomistic parts of the solution. For the coupling, it is important to satisfy macroscopic conservation laws such as mass and linear momentum as well as to prevent the artificial phase separation between the models of different resolutions which correspond to different free energies. Ideally, the all-atom resolution and the continuum part of the model should be smoothly connected so that the atomistic scales gradually transition to large continuum scales. Typically, a finite overlap region between MD and hydrodynamic part of the solution is needed to avoid sharp oscillations in density and pressure between different representations of the same chemical substance. ${ }^{24}$ To ensure smoothness of the transition from all-atom molecular dynamics to coarse-grained particles, the concept of particle scale bridging was introduced and implemented in the Adaptive Resolution Scheme (AdResS). ${ }^{24}$ In the subsequent work ${ }^{25}$, a concurrent triple scale model was suggested that combines the AdResS with Navier-Stokes equations through a hybrid molecular-continuum hydrodynamics scheme (Hybrid MD). Because of the flux coupling scheme, the concurrent AdResS-Hybrid MD method treats the multi-scale particle AdResS part and the continuum Navier-Stokes part as two different fields separated by an interface. However, it can be noted that such separation may not be ideal from the viewpoint of a sufficiently smooth transition from one part of the multiscale model to the other.

Following the state-variable coupling framework that avoids the need of an interface boundary, a twophase flow analogy method for smoothly coupling the Landau and Lifshitz Fluctuating Hydrodynamics equations (LL-FH) with MD was suggested in Ref. $^{26}$ and further developed in a series of subsequent works ${ }^{27,28}$. Rather than attempting to propose an ad-hoc modified Hamiltonian function to use it to subsequently derive the equations of motion of a hybrid molecular dynamics - continuum 
mechanics system, the analogy method considers a set of equations of motion of an atomistic-particle and a continuum representation of the same liquid.

Within the analogy approach, the equations of motion of hybrid molecular dynamics are treated as a "low-order" model for reducing the MD complexity to continuum hydrodynamics, which equations are defined in accordance with analogy to some physical macroscopic flow model of choice. In continuum fluid dynamics, this type of modelling approaches has been used for several decades. One notable example of these hybrid approaches is Lighthill's acoustic analogy method that was developed for modelling of sound generated by turbulence. ${ }^{29}$

In the current work, the hybrid model based on a hydrodynamic analogy with two-phase flows is adopted where the equations of motion are postulated as mass and momentum conservation laws of a nominally two-phase fluid. Concentration of each "phase", which would be the unknown variable in the conventional two-phase hydrodynamics model, is selected as a user-defined function of the analogy that controls resolution of the hybrid multiscale model. To avoid the artificial phase separation and preserve the continuity of variances of macroscopic density and velocity quantities across the different "phases", forcing terms are introduced as sources and sinks of the nominally two-phase flow equations without affecting the conservation of mass and momentum fluxes. The forcing terms are applied to force the MD part of the model to the continuum representation that is based on the Landau-Lifshitz Fluctuating Hydrodynamics (LL-FH) equations. ${ }^{30}$ The LL-FH equations use stochastic fluxes to model the effect of the Brownian motion at the smallest scale and reduce to the standard Navier-Stokes equations for large control volumes. For the discrete state of the fluid, the classical molecular dynamic equations are used at all-atom resolution. For simple molecular systems in $2 \mathrm{D}$, the hybrid method is shown to produce a converged solution that correctly captures macroscopic parameters such as standard deviation of thermal fluctuations and the mean flow velocity. ${ }^{26}$ This is achieved without introducing any ad-hoc treatment of MD particles such as "rescaling" of the MD solution or artificial "walls" and "barriers". In a further work ${ }^{31-33}$ of the authors a simplified, one-way coupled version of 
the original hybrid method was implemented for 3D simulations in GROMACS ${ }^{34,35}$, a popular opensource MD software. The implemented model assumes no feedback from the microscopic MD part of the solution on the macroscopic hydrodynamic part and also uses discrete particles in the entire computational domain so that the computational saving comes from skipping the calculation of particle-particle interactions in the hydrodynamic part of the solution domain. Despite these drawbacks, the model shows promise for simulations of a PCV2 virus capsid in water. In particular, the hybrid model is shown to correctly reproduce a stable capsid in a small computational domain and capture the relevant macroscopic transport characteristics of water and ions through the virus capsid compared to the reference all-atom simulation. On the model development front, recently, a triplescale one-way coupled scheme that combines the two-phase hydrodynamic analogy approach with multi-resolution molecular dynamics simulations (AdResS) is developed. ${ }^{36}$ By accounting for a smoother transition between the multi-atom water molecules to hydrodynamic particles in the flow, the triple-scale scheme is shown to lead to a reduced sensitivity to the model parameters while achieving an improved accuracy in test problems in comparison with the baseline MD-FH algorithm.

The one-way coupling assumption which ignores the feedback of the molecular scales on the continuum has been a significant simplification of the previous models based on the two-phase analogy method. Hence, in the current publication, a fully two-way coupled implementation of the method is developed and implemented for 3D simulations in GROMACS. Thanks to a consistent feedback effect from the discrete particles on the continuum flow, the molecular dynamics particles are localised in a small part of the continuum solution domain to drastically reduce the computational cost in comparison with the previous implementations. The two-way coupled formulation of the hybrid method that allows a seamless separation of the molecular dynamics particle zone and the pure continuum mechanics domain is a distinct novelty of the current hybrid multiscale model based on the two-phase flow analogy. 
The rest of the paper is organised as the following. In section 2, the governing two-phase analogy equations from Refs. ${ }^{26,37}$ are considered and new Generalised Landau-Lifshitz Fluctuating Hydrodynamics (GLL-FH) equations are derived. These equations can be viewed as an extension of the classical continuum LL-FH model in statistical mechanics to smoothly couple the continuum hydrodynamics with all-atom molecular dynamics simulations. The GLL-FH equations are formulated in a discrete form and in comparison with the classical LL-FH model include auxiliary time-dependent solution variables to describe the difference between the locally averaged fields of the molecular dynamics solution and the continuum hydrodynamics solution. In comparison with the standard LLFH model, the solution of the new generalised LL-FH equations converges to the locally averaged solution obtained on-the fly from all-atom molecular dynamics. Importantly, the locally averaged fields of the GLL-FH solution may not necessarily satisfy to the Gaussian statistics of the classical LLFH equations in the hybrid part of the model where $s<1$.

Similar to the classical LL-FH model, the GLL-FH equations are too complex for analytical solution, hence, a finite-volume computational scheme for solving these equations numerically is introduced in Appendix. In the computational implementation of these equations in GROMACS, the molecular dynamics calculation is limited to a small part of the computational domain while the GLL-FH equations are solved in the entire domain. For high-performance computing, the new scheme is implemented with the same vectorization approach as used in the original GROMACS algorithms. In section 4, numerical examples are provided for simulations of a liquid argon system at multiple resolution, which include the application of hydrodynamic forcing for Couette flow conditions and high-frequency acoustic wave propagation. 


\section{Theory}

\subsection{Conservation laws of the two-phase flow}

Following ${ }^{26}$, let us consider the computational domain filled with a nominally two-phase fluid (fig. 1), which contains a region of pure molecular dynamics (MD), a region of pure fluctuating hydrodynamics (FH), and a hybrid continuum-particle region (MD-FH). The two phases are a Lagrangian and an Eulerian representation of the same chemical substance. The phases are immersed into each other as 'fine grains', the surface tension effects are irrelevant, and both phases are assumed to simultaneously occupy the same control volume. The partial concentrations of the MD 'phase' and the pure hydrodynamic 'phase' are equal to $s$ and $1-s$, respectively, where $s$ is a parameter of the model, $0 \leq s \leq 1$. In general, $s$ is a user-defined scale function of space and time, which controls how much atomistic information is required in a particular region of the simulation domain.

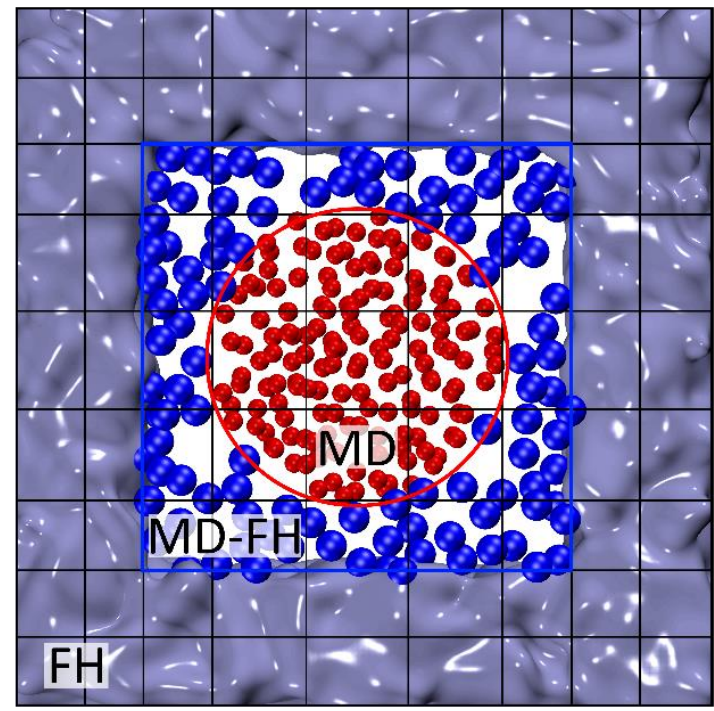

Fig. 1. A schematic of the hybrid particle-continuum computational domain which is divided into elementary control volumes $V$. 
Let us assume that this two-phase substance is isothermal and electrically neutral as well as corresponds to adiabatic heat ratio approaching unity. In this case, the mass and momentum equations of the continuum hydrodynamic equations can be solved separately from the energy equation. ${ }^{38}$ Then, following the standard approach in two-phase modelling, ${ }^{39}$ the dynamics of such two-phase system is governed by the conservation of mass of the continuum hydrodynamic (HD) "phase":

$$
\delta_{t}(s m)+\sum_{\gamma=1}^{6}\left(s \rho \tilde{\mathbf{u}} \cdot d \mathbf{n}^{\gamma}\right) \cdot \delta t=J_{1} \cdot \delta t
$$

and that of the mass of the molecular dynamic (MD) "phase":

$$
\delta_{t}\left(\sum_{p=1}^{N}\left[\left(1-s_{p}\right) m_{p}\right]\right)+\sum_{\gamma=1}^{6}\left(\sum_{p=1}^{N_{\gamma}}\left[\left(1-s_{p}\right) \rho_{p} \mathbf{u}_{p}\right] \cdot d \mathbf{n}^{\gamma}\right) \cdot \delta t=-J_{1} \cdot \delta t
$$

Here $m$ and $\rho=\frac{m}{V}$ are the mass and the density of the HD "phase", $m_{p}$ and $\rho_{p}=\frac{m_{p}}{V}$ are the particle mass and the effective particle density, $V$ is the cell volume, $N$ is the number of particles in the volume $V, \tilde{\mathbf{u}}$ is the velocity of mixture and $\mathbf{u}_{p}$ is the MD velocity of the particle $p, N_{\gamma}$ is the number of particles crossing the $\gamma$ th cell face with the normal $d \mathbf{n}^{\gamma}$ (which has the units of area), $s_{p}=s\left(x_{p}, y_{p}, z_{p}\right)$ is a particular value of the user-defined function $s$ in the location occupied by particle $p$ at time $t$.

The cell volume $V$ is defined as one of the non-overlapping elementary control volumes, or cells, which the hybrid computational domain is divided into (fig.1). The size of each cell is adjusted so that it contains a sufficient number of MD particles for statistical averaging, which for liquid argon simulations corresponds to about 100 atoms per control volume. ${ }^{37}$ It can be noted that in the limit of small macroscopic cell volumes, which at the same time should remain macroscopically large so that the particulate fields can be approximated by continuum distributions, the governing conservation laws 
(1) and (2) can be replaced by the corresponding system of partial-differential equations of two-phase flow type as discussed by Ref. ${ }^{26}$.

The cell-averaged value of the $s$-function is defined so that $s=\sum_{p=1}^{N}\left(s_{p} \rho_{p}\right) / \sum_{p=1}^{N} \rho_{p}$. For a linear reconstruction of the $s$-function inside the cell volume, it is equal to the $s$ value in the geometrical centre of the cell.

Finally, $J_{1}$ is the mass source/sink term which describes the transformation of mass between the "phases" and $\delta_{t}$ describes the change of a quantity over time $\delta t$.

Density of the two-phase mixture is defined so that:

$$
\tilde{\rho}=s \rho+\sum_{p=1}^{N}\left[\left(1-s_{p}\right) \rho_{p}\right]
$$

which expression can be re-arranged to:

$$
\tilde{\rho}=s \rho+\sum_{p=1}^{N} \rho_{p}-\sum_{p=1}^{N} s_{p} \rho_{p}=s \rho+\sum_{p=1}^{N} \rho_{p}-s \sum_{p=1}^{N} \rho_{p}=s \rho+(1-s) \sum_{p=1}^{N} \rho_{p} .
$$

Velocity of the mixture is defined with taking into account density averaging so that:

$$
\tilde{u}_{i}=\left[s \rho u_{i}+\sum_{p=1}^{N}\left[\left(1-s_{p}\right) \rho_{p} u_{i p}\right]\right] / \tilde{\rho}
$$

The momentum equation of the continuum hydrodynamic "phase" is considered next:

$$
\delta_{t}\left(s m u_{i}\right)+\sum_{\gamma=1}^{6}\left(s \rho u_{i} \tilde{\mathbf{u}} \cdot d \mathbf{n}^{\gamma}\right) \cdot \delta t=s F_{i} \cdot V \delta t+J_{2} \cdot \delta t
$$

where $F_{i}$ represents the stress per unit volume, which includes the deterministic and the stochastic parts:

$$
F_{i}=\bar{F}_{i}+\tilde{F}_{i}
$$


The force, $F_{i}$ is directly related to the stress tensor in accordance with the standard continuum mechanics relation, $F_{i}=\nabla_{j}\left(\bar{\Pi}_{i j}+\tilde{\Pi}_{i j}\right)$, and the stresses $\bar{\Pi}_{i j}, \tilde{\Pi}_{i j}$ are modelled in accordance with the Landau Lifshitz Fluctuating Hydrodynamic Navier-Stokes equations, ${ }^{30}$ which are briefly summarised below:

$$
\begin{aligned}
& \frac{\partial \rho}{\partial t}+\operatorname{div}(\rho \mathbf{u})=0, \\
& \frac{\partial\left(\rho u_{i}\right)}{\partial t}+\operatorname{div}\left(\rho u_{i} \mathbf{u}\right)=\nabla_{j}\left(\bar{\Pi}_{i j}+\tilde{\Pi}_{i j}\right)
\end{aligned}
$$

Here $p=p(\rho)$ in accordance with the isothermal fluid equation of state, the stress tensor $\bar{\Pi}_{i j}$ and its fluctuating component $\tilde{\Pi}_{i j}$ are defined so that

$$
\begin{aligned}
& \bar{\Pi}_{i j}=-(p-\zeta \operatorname{div} \mathbf{u}) \delta_{i j}+\eta\left(\partial_{i} u_{j}+\partial_{i} u_{j}-2 D^{-1} \operatorname{div} \mathbf{u} \delta_{i j}\right), \\
& \tilde{\Pi}_{i j}=\zeta \operatorname{div} \tilde{\mathbf{u}} \delta_{i j}+\eta\left(\partial_{i} \tilde{u}_{j}+\partial_{i} \tilde{u}_{j}-2 D^{-1} \operatorname{div} \tilde{\mathbf{u}} \delta_{i j}\right) .
\end{aligned}
$$

Here $\eta$ and $\zeta$ are shear and bulk viscosity coefficients. $\tilde{\Pi}_{i j}$ is modelled as a random Gaussian matrix with zero mean and covariance:

$$
\left\langle\tilde{\Pi}_{i j}\left(\mathbf{r}_{1}, t_{1}\right) \tilde{\Pi}_{k l}\left(\mathbf{r}_{2}, t_{2}\right)\right\rangle=2 k_{B} T\left[\eta\left(\delta_{i k} \delta_{j l}+\delta_{i l} \delta_{j k}\right)+\left(\zeta-2 D^{-1} \eta\right) \delta_{i j} \delta_{k l}\right] \delta\left(t_{1}-t_{2}\right) \delta\left(\mathbf{r}_{1}-\mathbf{r}_{2}\right) .
$$

Following Ref..$^{30}$, the stochastic stress tensor is represented explicitly so that

$$
\tilde{\Pi}_{i j} \cong \sqrt{\frac{2 k_{B} T}{\delta t \delta V}}\left(\sqrt{2} \sqrt{\eta} \cdot G_{i j}^{S}+\sqrt{D} \sqrt{\zeta} \cdot \operatorname{tr}[\mathbf{G}] \cdot E_{i j} / D\right)
$$

where $\mathbf{G}$ is a random Gaussian matrix with zero mean and covariance $\left\langle G_{i j} G_{k l}\right\rangle=\delta_{i j} \delta_{k l}$, $G_{i j}^{S}=\frac{G_{i j}+G_{i j}^{T}}{2}-\operatorname{tr}[\mathbf{G}] \cdot E_{i j} / D$, is a random symmetric matrix with zero trace, $\mathbf{E}$ is the identity matrix, and $\operatorname{tr}[\mathbf{G}]$ is the trace of the matrix $\mathbf{G}$. 
It can be noted that the LL-FH equations (6)-(9) are a limiting case of discrete conservation laws Eqs.(1)-(5) for a sufficiently large control volume in case $s$ equal to 1.

The equation for momentum of the MD "phase" is considered next:

$$
\delta_{t}\left(\sum_{p=1}^{N}\left[\left(1-s_{p}\right) m_{p} u_{i p}\right]\right)+\sum_{\gamma=1}^{6}\left(\sum_{p=1}^{N_{\gamma}}\left[\left(1-s_{p}\right) \rho_{p} u_{i p} \mathbf{u}_{p}\right] \cdot d \mathbf{n}^{\gamma}\right) \delta t=\sum_{p=1}^{N}\left[\left(1-s_{p}\right) F_{i p}^{M D}\right] \cdot V \delta t-J_{2} \delta t
$$

From comparing Eqs.(1),(2) and Eqs.(5),(10), it can be seen that the two-phase fluid system preserves conservation fluxes of mass and momentum. That is, the two-phase model leads to the conservation of mass and momentum in case of no external forces applied in the system.

The above two-phase analogy equations are written in a compact form which is useful for understanding of physical flow analogy behind this hybrid model. However, this formulation is not most suitable for a practical implementation since equations (1),(2),(5), and (10) are not closed. They contain unknown variables such as the particle fields as well as the forcing source and sink terms which are required to smoothly transition from one representation to another. Moreover, the $s$ and $(1-s)$ functions inside the time derivatives and fluxes of these equations tend to zero in the liming cases of $s=0$ or $s=1$, which makes the corresponding parts of the equations to degenerate. For example, in the continuum limit of very large control volumes where a partial-differential equations description apply, this would be equivalent to a change of the equation type from parabolic to elliptic, which is not ideal from the viewpoint of numerical solution. Hence, in the following sections the governing system of equations (1)-(10) will be re-arranged to a more suitable form which will also include an appropriate differential constraint to prevent the "phase" separation.

\subsection{Differential constrains to prevent the phase separation}

To prevent the phase separation, a phase mixing, or binding, process is imposed in accordance with the forcing source and sink terms in the analogy equations (1),(2),(5), and (10). For efficiency, the 
forcing terms implicitly include the difference between the molecular dynamic "phase" and the continuum fields.

The forcing terms can be determined a-posteriori so that the difference between the molecular dynamic "phase" and the mixture field decays in time exponentially:

$$
\frac{D}{D t} \rho^{\prime}=-\alpha \cdot f(s) \rho^{\prime} \text { and } \frac{D}{D t} q_{i}^{\prime}=-\beta \cdot f(s) q_{i}^{\prime}
$$

where $\alpha$ and $\beta$ are some positive relaxation parameters, $D / D t$ is the material derivative with a conservative convective part, and the differences between the molecular dynamics and continuum fields are defined so that

$$
\rho^{\prime}=\tilde{\rho}-\sum_{p=1}^{N} \rho_{p}
$$

and

$$
q_{i}^{\prime}=\tilde{\rho} \tilde{u}_{i}-\sum_{p=1}^{N} \rho_{p} u_{i p}
$$

Here $f(s)$ is a user-defined function defining the decay rate of the differences so that it is equal to 0 in the MD zone $(s=0)$ and tends to a constant in the continuum region $(s=1)$ so that the difference between the two phases decays to zero with time, for example:

$$
f(s)=s(1-s)+\alpha_{0} s, \quad \alpha_{0}>0
$$

A numerical method for solving equations (11) will be discussed in Section 3 with the computational scheme details being provided in Appendix of the article.

Let us now consider the following modified MD equations:

$$
\frac{d x_{i p}}{d t}=\left(1-s_{p}\right) u_{i p}+s_{p} \tilde{u}_{i p}-\alpha \cdot \int_{x_{0 i}}^{x_{i}}\left(f(s) \rho^{\prime} / \sum_{p=1, N} \rho_{p}\right) d y_{i},
$$


where $x_{i p}$ is the $i$-th coordinate component of particle $p$, whose momentum equation is given by

$$
\begin{aligned}
& \frac{d u_{i p}}{d t}=\left(1-s_{p}\right) F_{i p}{ }^{M D} / \rho_{p}+s_{p} F_{i p} / \sum_{p=1, N} \rho_{p}+\beta \cdot f\left(s_{p}\right) \cdot q_{i}^{\prime} / \sum_{p=1, N} \rho_{p}- \\
& -\frac{1}{V} \sum_{\gamma=1}^{6}\left(\sum_{p=1, N} \alpha u_{i p} \rho_{p}\left[\int_{x_{0 j}}^{x_{j}}\left(f(s) \rho^{\prime} / \sum_{p=1, N} \rho_{p}\right) d y_{j}\right]\right) d n_{j}^{\gamma} / \sum_{p=1, N} \rho_{p}
\end{aligned}
$$

For a sufficiently large control volume, the last equation can be written in the equivalent differential form as

$$
\begin{aligned}
& \frac{d u_{i p}}{d t}=\left(1-s_{p}\right) F_{i p}^{M D} / \rho_{p}+s_{p} F_{i p} / \sum_{p=1, N} \rho_{p}+\beta \cdot f\left(s_{p}\right) \cdot q_{i}^{\prime} / \sum_{p=1, N} \rho_{p}- \\
& -\left(\frac{\partial}{\partial x_{j}}\left(\sum_{p=1, N} \alpha u_{i p} \rho_{p}\left[\int_{x_{0 j}}^{x_{j}}\left(f(s) \rho^{\prime} / \sum_{p=1, N} \rho_{p}\right) d y_{j}\right]\right)\right) / \sum_{p=1, N} \rho_{p}
\end{aligned}
$$

where $\mathbf{x}_{0}$ is the centre of mass (or geometrical centre) of the pure MD zone $(s=0)$. The integral is assumed to be taken over the particle/continuum zone. The latter zone includes the pure MD region and the boundaries that separates particles from continuum where $s \sim 1$ and a periodic boundary condition is applied for particles.

The above equations (14) and (15) describe the dynamics of MD particles in the presence of a collective hydrodynamic field, which, in turn, is affected by the particles, as it will be discussed in detail in the following sections.

It should be remarked that the above modified MD particle velocity equation (15), which corresponds to a modification of Newton's second law for particle interactions by addition of some effective “coarse-grained” force, resembles many existing coarse-grained MD formulations. For example, it can be compared with the constrained particle dynamics, Non-Equilibrium MD (NEMD) methods as well as with the AdResS method, ${ }^{24}$ which was already mentioned in the introduction, and which also uses some weighting function for a linear interpolation of the force field in the hybrid zone. 
Despite the apparent similarity, the role of the $s$-function in the suggested two-phase analogy method, which provides controllers of density and momentum in Eqs (14) and (15), is different. Indeed, an important difference of the present hybrid approach from the existing hybrid MD methods in the literature is the strict conservation of mass and momentum in the entire multiscale system that includes not only the two limiting MD and continuum hydrodynamics states but also the hybrid zone which connects the two limits. To achieve such consistency without any local violation of the governing conservation laws, the $s$-function is not a just a linear interpolation parameter for the MD force but is an active variable that is involved in the micro-macroscopic dynamics of the two-phase flow analogy equations. Because of local conservation of mass and momentum fluxes of the two-phase mixture in the entire range of phase "concentrations" including the intermediate region, $0<s<1$, macroscopic properties of the governing continuum equations, including those of acoustic wave propagation such as the linear dispersion relation, are preserved.

The forcing source and sink functions of the two-phase analogy method are defined in such a way so that the local conservation of mass and momenta is not violated at any point. The effect of the forcing functions can be compared with the effect of the external boundary force that accounts for the virial component of the pressure as suggested by Ref. ${ }^{40}$ and also with the effect of the thermodynamic force of the AdResS model. For example, the AdResS thermodynamic force preserves continuity of pressure across the hybrid multi-resolution particle zone. But this preservation does not include continuity of the pressure gradient that determines acoustic sound speed, hence, does not support the same acoustic wave dispersion relation across the hybrid zone in this case.

The suggested way of smoothly connecting continuum hydrodynamics with particles in the two-phase analogy framework can also be compared with the Hybrid MD model. ${ }^{25}$ The latter is based around the idea of the momentum flux exchange between the MD and the Navier-Stokes model at the hybrid interface $H$ for the conservation of mass and momentum fluxes. However, the Hybrid MD model still permits local violations of mass and momentum in the buffer domain $B$, which connects $H$ and MD 
zones and which is needed to operate the two zones without numerical artefacts. $B$ is a mass and momentum reservoir of the particles and used for imposing the external momentum into the molecular dynamic region using a particle insertion-deletion-sorting algorithm based on the energy minimization. ${ }^{41}$ This reservoir plays the role of an artificial interface between the continuum and discrete particle representations of the same chemical substance: the models can inform one another in terms of the transport coefficients and other macroscopic properties but remain very different conceptually. This is not the case in the suggested two-phase flow analogy model where the MD and the hydrodynamic representation of the same chemical substance converge one to another in accordance with the conservation laws and avoiding any need for external reservoirs of mass and momentum.

As discussed above, the $s$-function of the two-phase flow analogy method plays an important role in a consistent definition of mass and momentum transfer between the two "phases" in accordance with the governing conservation laws (1),(2),(5), (10). Having defined the modified MD particle equations (14) and (15), we will now demonstrate how they are consistent with the governing conservation laws of the two-phase liquid. This demonstration will also help deriving an equivalent formulation of the governing equations that can be suitable for a practical numerical implementation. The resulting formulation will be called Generalised Landau-Lifshitz Fluctuating Hydrodynamics equations.

First, let us derive some expressions which will be used in further analysis. From (12) we obtain

$$
\tilde{\rho}=\rho^{\prime}+\sum_{p=1}^{N} \rho_{p}
$$

and from (13)

$$
\tilde{u}_{i}=\left(q_{i}^{\prime}+\sum_{p=1}^{N} u_{i p} \rho_{p}\right) /\left(\rho^{\prime}+\sum_{p=1}^{N} \rho_{p}\right)=\left(q_{i}^{\prime}+\sum_{p=1}^{N} u_{i p} \rho_{p}\right) / \tilde{\rho},
$$

Let us consider a particle mass conservation law in control volume $V$ : 


$$
\frac{\delta_{t}\left(\sum_{p=1}^{N} m_{p}\right)}{\delta t}+\sum_{\gamma=1}^{6}\left[\left(\sum_{p=1}^{N_{\gamma}} \frac{d \mathbf{x}_{p}}{d t} \rho_{p}\right) d \mathbf{n}^{\gamma}\right]=0 .
$$

By substituting $\sum_{p=1}^{N} \rho_{p}=\tilde{\rho}-\rho^{\prime}$ and (14) into the particle conservation law, one obtains:

$$
\frac{\delta_{t}\left(\tilde{\rho}-\rho^{\prime}\right)}{\delta t}+\frac{1}{V} \sum_{\gamma=1}^{6}\left[\left(\sum_{p=1}^{N_{\gamma}}\left[\left(1-s_{p}\right) u_{i p} \rho_{p}+s_{p} \tilde{u}_{i p} \rho_{p}-\alpha \rho_{p} \int_{x_{0 i}}^{x_{i}}\left(f(s) \rho^{\prime} / \sum_{p=1, N} \rho_{p}\right) d y_{i}\right]\right) d n_{i}^{\gamma}\right]=0
$$

By rearranging, the last equation becomes:

$$
\frac{\delta_{t}\left(\tilde{\rho}-\rho^{\prime}\right)}{\delta t}+\frac{1}{V} \sum_{\gamma=1}^{6}\left[\left(\sum_{p=1}^{N_{\gamma}} s_{p} \tilde{u}_{i p} \rho_{p}\right) d n_{i}^{\gamma}\right]=-\frac{1}{V} \sum_{\gamma=1}^{6}\left[\left(\sum_{p=1}^{N_{\gamma}}\left(1-s_{p}\right) u_{i p} \rho_{p}\right) d n_{i}^{\gamma}\right]+\alpha f(s) \rho^{\prime}
$$

which, in turn, can be reduced

$$
\frac{\delta_{t}\left(\tilde{\rho}-\rho^{\prime}\right)}{\delta t}+\frac{1}{V} \sum_{\gamma=1}^{6}\left[\left(s \tilde{u}_{i} \sum_{p=1}^{N} \rho_{p}\right) d n_{i}^{\gamma}\right]=Q_{\rho}+\alpha f(s) \rho^{\prime}
$$

where

$$
Q_{\rho}=-\frac{1}{V} \sum_{\gamma=1}^{6}\left[\left(\sum_{p=1}^{N_{\gamma}}\left(1-s_{p}\right) u_{i p} \rho_{p}\right) d n_{i}^{\gamma}\right]
$$

is the effective density source due to the MD "phase" and $s \tilde{u}_{i}$ is an averaged product of the $i$-th velocity component of the two-phase mixture and the $s$-function for each face $\gamma$ of the control volume $V$.

By introducing a new dependent variable, $\rho^{*}=\tilde{\rho}-\rho^{\prime}$ the conservation law for the continuum field $\rho^{*}$ is obtained:

$$
\frac{\delta_{t} \rho^{*}}{\delta t}+\frac{1}{V} \sum_{\gamma=1}^{6}\left[s \rho^{*} \tilde{u}_{i} d n_{i}^{\gamma}\right]=Q_{\rho}+\alpha f(s) \rho^{\prime},
$$

which, assuming a sufficiently large control volume, can be written in a differential form: 


$$
\frac{\partial}{\partial t} \rho^{*}+\frac{\partial}{\partial x_{i}} s \tilde{u}_{i} \rho^{*}=Q_{\rho}+\alpha f(s) \rho^{\prime}
$$

Having computed $\rho^{*}$ from (19), the mixture density field $\tilde{\rho}$ can be determined as $\tilde{\rho}=\rho^{*}+\rho^{\prime}$, where $\rho^{\prime}$ is obtained from solving (11).

It can be noted that in comparison with the "phase" density equations (1) and (2), the new Eq.(19) is simpler to solve as it does not contain a vanishing parameter $s$ or $(1-s)$ inside the time derivative term. At this point we still need to show how Eq.(19) amounts to exactly the same mass conservation law in accordance with the governing conservations laws of Section 2.1. This is what is has been addressed below:

By adding $-\delta_{t} \sum_{p=1}^{N} s_{p} m_{p}=-\delta_{t} \sum_{p=1}^{N} s_{p} m_{p}$ to Eq.(18) and re-arranging the result, the following expression is obtained:

$$
\begin{aligned}
& \delta_{t} \sum_{p=1}^{N}\left[\left(1-s_{p}\right) m_{p}\right]+\sum_{\gamma=1}^{6}\left[\left(\sum_{p=1}^{N_{\gamma}}\left(1-s_{p}\right) u_{i p} \rho_{p}\right) d n_{i}^{\gamma}\right] \delta t= \\
& =-\delta_{t} \sum_{p=1}^{N} s_{p} m_{p}-\sum_{\gamma=1}^{6}\left[\left(\sum_{p=1}^{N_{\gamma}} s_{p} \tilde{u}_{i p} \rho_{p}\right) d n_{i}^{\gamma}\right] \delta t+\alpha f(s) \rho^{\prime} V \delta t
\end{aligned}
$$

By comparing (20) and (2), the definition of the source sink/source function follows:

$$
J_{1}=\frac{\delta_{t} \sum_{p=1}^{N} s_{p} m_{p}}{\delta t}+\sum_{\gamma=1}^{6}\left[\left(\sum_{p=1}^{N_{\gamma}} s_{p} \tilde{u}_{i p} \rho_{p}\right) d n_{i}^{\gamma}\right]-\alpha f(s) \rho^{\prime} V
$$

To show consistency of the derived sink/source function with the continuum phase equation (1), let us start from the equation for the difference between the molecular dynamics and the continuum field, $\frac{D}{D t} \rho^{\prime}=-\alpha \cdot f(s) \rho^{\prime}$, which in the integral form reads: 


$$
\delta_{t}\left(\rho^{\prime}\right)+\frac{1}{V} \sum_{\gamma=1}^{6}\left(\rho^{\prime} \tilde{\mathbf{u}} \cdot d \mathbf{n}^{\gamma}\right) \cdot \delta t=-\alpha \cdot f(s) \rho^{\prime} \cdot \delta t
$$

By re-arranging this equation to

$$
\begin{aligned}
& \delta_{t}\left(\tilde{\rho}-\sum_{p=1, N}\left(1-s_{p}\right) \rho_{p}-\sum_{p=1, N} s_{p} \rho_{p}\right)+\frac{1}{V} \sum_{\gamma=1}^{6}\left(\left[\tilde{\mathbf{u}} \tilde{\rho}-\tilde{\mathbf{u}} \sum_{p=1, N}\left(1-s_{p}\right) \rho_{p}-\sum_{p=1, N} \tilde{\mathbf{u}}_{p} s_{p} \rho_{p}\right] \cdot d \mathbf{n}^{\gamma}\right) \cdot \delta t= \\
& =-\alpha \cdot f(s) \rho^{\prime} \cdot \delta t
\end{aligned}
$$

and introducing a new dependent variable corresponding to the continuum phase with using Eq.(3), the following balace law is obtained

$$
\delta_{t}\left(s \rho-\sum_{p=1, N} s_{p} \rho_{p}\right)+\frac{1}{V} \sum_{\gamma=1}^{6}\left(\left[\tilde{\mathbf{u}} s \rho-\sum_{p=1, N} \tilde{\mathbf{u}}_{p} s_{p} \rho_{p}\right] \cdot d \mathbf{n}^{\gamma}\right) \cdot \delta t=-\alpha \cdot f(s) \rho^{\prime} \cdot \delta t
$$

The last equation is equivalent to Eq.(1), where the source function is defined in accordance with equation (21):

$$
\delta_{t}(s m)+\sum_{\gamma=1}^{6}\left(s \rho \tilde{\mathbf{u}} \cdot d \mathbf{n}^{\gamma}\right) \cdot \delta t=\delta_{t}\left(\sum_{p=1, N} s_{p} m_{p}\right)+\sum_{\gamma=1}^{6}\left(\left[\sum_{p=1, N} \tilde{\mathbf{u}}_{p} s_{p} \rho_{p}\right] \cdot d \mathbf{n}^{\gamma}\right) \cdot \delta t-\alpha f(s) \rho^{\prime} V \cdot \delta t \equiv J_{1} \cdot \delta t
$$

To complete the formulation, let us now derive the corresponding momentum equations.

By starting from the equation of particle momentum balance:

$$
\delta_{t}\left(\sum_{p=1}^{N} m_{p} u_{i p}\right)+\sum_{\gamma=1}^{6}\left[\left(\sum_{p=1}^{N_{\gamma}} \frac{d \mathbf{x}_{p}}{d t} \rho_{p} u_{i p}\right) d \mathbf{n}^{\gamma}\right] \cdot \delta t=\sum_{p=1}^{N}\left[m_{p} \frac{d u_{i p}}{d t}\right] \cdot \delta t
$$

By using $\sum_{p=1}^{N} u_{i p} \rho_{p}=\tilde{u}_{i} \tilde{\rho}-q_{i}^{\prime}$ and (14),(15) the momentum balance equation is re-arranged to: 


$$
\begin{aligned}
& \delta_{t}\left(\tilde{u}_{i} \tilde{\rho}-q_{i}^{\prime}\right)+\frac{1}{V} \sum_{\gamma=1}^{6}\left[\sum_{p=1}^{N_{\gamma}}\left(\left(1-s_{p}\right) u_{j p} \rho_{p} u_{i p}+s_{p} \tilde{u}_{j p} \rho_{p} u_{i p}-\alpha \cdot \rho_{p} u_{i p} \int_{x_{0 j}}^{x_{j}}\left(f(s) \rho^{\prime} / \sum_{p=1, N} \rho_{p}\right) d y_{j}\right) d n_{j}^{\gamma}\right] \cdot \delta t= \\
& =\frac{1}{V} \sum_{p=1}^{N}\left[\begin{array}{l}
\left.m_{p}\left(1-s_{p}\right) F_{i p}^{M D} / \rho_{p}+m_{p} s_{p} F_{i p} / \sum_{p=1, N} \rho_{p}+m_{p} \beta \cdot f\left(s_{p}\right) \cdot q_{i}^{\prime} / \sum_{p=1, N} \rho_{p}-\right] \\
\left.-\rho_{p} \sum_{\gamma=1}^{6}\left(\sum_{p=1, N} \alpha u_{i p} \rho_{p}\left[\int_{x_{0 j}}^{x_{j}}\left(f(s) \rho^{\prime} / \sum_{p=1, N} \rho_{p}\right) d y_{j}\right]\right) d n_{j}^{\gamma} / \sum_{p=1, N} \rho_{p}\right] \cdot \delta t
\end{array}\right]
\end{aligned}
$$

which becomes

$$
\begin{aligned}
& \delta_{t}\left(\tilde{u}_{i} \tilde{\rho}-q_{i}^{\prime}\right)+\frac{1}{V} \sum_{\gamma=1}^{6}\left[\sum_{p=1}^{N_{\gamma}}\left(\left(1-s_{p}\right) u_{j p} \rho_{p} u_{i p}+s_{p} \tilde{u}_{j p} \rho_{p} u_{i p}\right) d n_{j}^{\gamma}\right] \cdot \delta t= \\
& =\frac{1}{V} \sum_{p=1}^{N}\left[m_{p}\left(1-s_{p}\right) F_{i p}^{M D} / \rho_{p}+m_{p} s_{p} F_{i p} / \sum_{p=1, N} \rho_{p}+m_{p} \beta f\left(s_{p}\right) \cdot q_{i}^{\prime} / \sum_{p=1, N} \rho_{p}\right] \cdot \delta t
\end{aligned}
$$

Here it was assumed that

$$
\begin{aligned}
& \sum_{\gamma=1}^{6}\left[\sum_{p=1}^{N_{\gamma}}\left(-\alpha u_{i p} \rho_{p} \int_{x_{0 j}}^{x_{j}}\left(f(s) \rho^{\prime} / \sum_{p=1, N} \rho_{p}\right) d y_{j}\right) d n_{j}^{\gamma}\right] \approx \\
& \approx \sum_{p=1}^{N}\left[-\rho_{p} \sum_{\gamma=1}^{6}\left(\sum_{p=1, N} \alpha u_{i p} \rho_{p}\left[\int_{x_{0 j}}^{x_{j}}\left(f(s) \rho^{\prime} / \sum_{p=1, N} \rho_{p}\right) d y_{j}\right]\right) d n_{j}^{\gamma} / \sum_{p=1, N} \rho_{p}\right]
\end{aligned}
$$

since the expression on the left-hand-side of the above equation can be approximated by the corresponding cell-volume average, which is then identical to the right-hand-side. Indeed, the righthand-side corresponds to the same cell-volume average that is multiplied by the "density" of each atom in control volume, $\rho_{p}$, summed over all particles, and then divided by the collective density of the atoms (the density of liquid per control volume).

By defining a new dependent variable quantity, $q_{i}=\tilde{u}_{i} \tilde{\rho}-q_{i}^{\prime}$ and rearranging the corresponding prognostic equation for this quantity, the following momentum balance equation is obtained: 


$$
\begin{aligned}
& \delta_{t} q_{i}+\frac{1}{V} \sum_{\gamma=1}^{6}\left[\sum_{p=1}^{N_{\gamma}}\left(\left(1-s_{p}\right) u_{j p} \rho_{p} u_{i p}\right) d n_{j}^{\gamma}\right] \cdot \delta t+\frac{1}{V} \sum_{\gamma=1}^{6}\left[\sum_{p=1}^{N_{\gamma}}\left(s_{p} \tilde{u}_{j p} \rho_{p} u_{i p}\right) d n_{j}^{\gamma}\right] \cdot \delta t= \\
& =\sum_{p=1}^{N}\left[\left(1-s_{p}\right) F_{i p}{ }^{M D}\right] \cdot \delta t+s_{p} F_{i p} \cdot \delta t+\sum_{p=1}^{N}\left[\rho_{p} \beta f\left(s_{p}\right) \cdot q_{i}^{\prime} / \sum_{p=1, N} \rho_{p}\right] \cdot \delta t
\end{aligned}
$$

For an appropriate reconstruction of the averages inside the last summation, this leads to

$$
\begin{aligned}
& \delta_{t} q_{i}+\frac{1}{V} \sum_{\gamma=1}^{6}\left[\sum_{p=1}^{N_{\gamma}}\left(s_{p} \tilde{u}_{j p} \rho_{p} u_{i p}\right) d n_{j}^{\gamma}\right] \cdot \delta t= \\
& =\sum_{p=1}^{N}\left[\left(1-s_{p}\right) F_{i p}^{M D}\right] \cdot \delta t-\frac{1}{V} \sum_{\gamma=1}^{6}\left[\sum_{p=1}^{N_{\gamma}}\left(\left(1-s_{p}\right) u_{j p} \rho_{p} u_{i p}\right) d n_{j}^{\gamma}\right] \cdot \delta t+s F_{i} \cdot \delta t+\beta f(s) \cdot q_{i}^{\prime} \cdot \delta t
\end{aligned}
$$

and introducing averages for each face of the control volume, this reduces to

$$
\frac{\delta_{t} q_{i}}{\delta t}+\frac{1}{V} \sum_{\gamma=1}^{6}\left[s \tilde{u}_{j} \sum_{p=1}^{N}\left(\rho_{p} u_{i p}\right) d n_{j}^{\gamma}\right]=Q_{i}+s F_{i}+\beta f(s) \cdot q_{i}^{\prime},
$$

where the effective source

$$
Q_{i}=\sum_{p=1}^{N}\left[\left(1-s_{p}\right) F_{i p}{ }^{M D}\right]-\frac{1}{V} \sum_{\gamma=1}^{6}\left[\sum_{p=1}^{N_{\gamma}}\left(\left(1-s_{p}\right) u_{j p} \rho_{p} u_{i p}\right) d n_{j}^{\gamma}\right]
$$

corresponds to the contribution to the force and the momentum difference from the MD particles and $s \tilde{u}_{j}$ is an averaged product of the $j$-th velocity component of the two-phase mixture and the $s$-function for each face $\gamma$ of the control volume $V$.

By recalling $\sum_{p=1}^{N} u_{i p} \rho_{p}=\tilde{u}_{i} \tilde{\rho}-q_{i}^{\prime}=q_{i}$, the last equation is re-arranged to

$$
\frac{\delta_{t} q_{i}}{\delta t}+\frac{1}{V} \sum_{\gamma=1}^{6}\left[s q_{i} \tilde{u}_{j} d n_{j}^{\gamma}\right]=Q_{i}+s F_{i}+\beta f(s) \cdot q_{i}^{\prime}
$$

which, for a sufficiently large control volume, can be represented in the equivalent differential form as 


$$
\frac{\partial q_{i}}{\partial t}+\frac{\partial}{\partial x_{j}}\left(s \tilde{u}_{j} q_{i}\right)=Q_{i}+s F_{i}+\beta f(s) \cdot q_{i}^{\prime}
$$

Having determined $q_{i}$ is from (23), the two-phase mixture velocity $\tilde{u}_{i}$ is calculated as $\tilde{u}_{i}=\left(q_{i}+q_{i}^{\prime}\right) / \tilde{\rho}$ , where $q_{i}^{\prime}$ is obtained from (11) as discussed in the numerical implementation section.

It can be noted that in comparison with the "phase" density equations (5) and (10), Eq(23) is simpler to solve because it does not contain a vanishing parameter, $s$ or $(1-s)$ inside the time derivative term. For completeness, we now need to show how $\mathrm{Eq}(23)$ is equivalent to the same momentum flux conservation law in accordance with the analogy equations Eq.(5) and (10).

By adding $-\delta_{t} \sum_{p=1}^{N} s_{p} m_{p} u_{i p}=-\delta_{t} \sum_{p=1}^{N} s_{p} m_{p} u_{i p}$ to Eq.(22) and rearranging the result, the following equation for the momentum is obtained:

$$
\begin{aligned}
& \delta_{t}\left(\sum_{p=1}^{N}\left(1-s_{p}\right) m_{p} u_{i p}\right)+\sum_{\gamma=1}^{6}\left[\sum_{p=1}^{N_{\gamma}}\left(\left(1-s_{p}\right) u_{j p} \rho_{p} u_{i p}\right) d n_{j}^{\gamma}\right] \cdot \delta t=\sum_{p=1}^{N}\left[\left(1-s_{p}\right) F_{i p}{ }^{M D}\right] \cdot V \delta t- \\
& -\delta_{t} \sum_{p=1}^{N} s_{p} m_{p} u_{i p}-\sum_{\gamma=1}^{6}\left[\sum_{p=1}^{N_{\gamma}}\left(s_{p} \tilde{u}_{j p} \rho_{p} u_{i p}\right) d n_{j}^{\gamma}\right] \cdot \delta t+s F_{i} \cdot V \delta t+\beta f(s) \cdot q_{i}^{\prime} \cdot V \delta t
\end{aligned}
$$

By comparing the above equation with Eq.(10), the corresponding momentum source/sink term is determined:

$$
J_{2}=\frac{\delta_{t} \sum_{p=1}^{N} s_{p} m_{p} u_{i p}}{\delta t}+\sum_{\gamma=1}^{6}\left[\sum_{p=1}^{N_{\gamma}}\left(s_{p} \tilde{u}_{j p} \rho_{p} u_{i p}\right) d n_{j}^{\gamma}\right]-s F_{i} \cdot V-\beta f(s) \cdot q_{i}^{\prime} \cdot V
$$

To demonstrate how this expression is consistent with the continuum phase equation (5), let us consider the equation defining the evolution of the difference between the molecular dynamics and continuum field $\frac{D}{D t} q_{i}^{\prime}=-\beta \cdot f(s) q_{i}^{\prime}$, which in the integral form is given by: 


$$
\delta_{t}\left(q_{i}^{\prime}\right)+\frac{1}{V} \sum_{\gamma=1}^{6}\left(q_{i}^{\prime} \tilde{\mathbf{u}} \cdot d \mathbf{n}^{\gamma}\right) \cdot \delta t=-\beta \cdot f(s) q_{i}^{\prime} \cdot \delta t
$$

After some re-arrangement, the last equation becomes:

$$
\delta_{t}\left(\tilde{\rho} \tilde{u}_{i}-\sum_{p=1, N} \rho_{p} u_{i p}\right)+\frac{1}{V} \sum_{\gamma=1}^{6}\left(\left[\tilde{\mathbf{u}} \tilde{\rho} \tilde{u}_{i}-\tilde{\mathbf{u}} \sum_{p=1, N} \rho_{p} u_{i p}\right] \cdot d \mathbf{n}^{\gamma}\right) \cdot \delta t=-\beta \cdot f(s) q_{i}^{\prime} \cdot \delta t
$$

By substituting the definition of the mixture velocity, $\tilde{u}_{i}=\left[s \rho u_{i}+\sum_{p=1}^{N}\left[\left(1-s_{p}\right) \rho_{p} u_{i p}\right]\right] / \tilde{\rho}$, the above expression is further rearranged to:

$$
\delta_{t}\left(s \rho u_{i}-\sum_{p=1, N} s_{p} \rho_{p} u_{i p}\right)+\frac{1}{V} \sum_{\gamma=1}^{6}\left(\left[s \tilde{\mathbf{u}} \rho u_{i}-\tilde{\mathbf{u}} \sum_{p=1, N} s_{p} \rho_{p} u_{i p}\right] \cdot d \mathbf{n}^{\gamma}\right) \cdot \delta t=-\beta \cdot f(s) q_{i}^{\prime} \cdot \delta t
$$

which is equivalent to

$$
\begin{aligned}
& \delta_{t}\left(s m u_{i}\right)+\sum_{\gamma=1}^{6}\left(s \rho u_{i} \tilde{\mathbf{u}} \cdot d \mathbf{n}^{\gamma}\right) \cdot \delta t= \\
& =\delta_{t}\left(\sum_{p=1, N} s_{p} m_{p} u_{i p}\right)+\sum_{\gamma=1}^{6}\left(\left[\tilde{\mathbf{u}} \sum_{p=1, N} s_{p} \rho_{p} u_{i p}\right] \cdot d \mathbf{n}^{\gamma}\right) \cdot \delta t-\beta \cdot f(s) q_{i}^{\prime} \cdot V \delta t \equiv J_{2} \cdot \delta t+s F_{i} \cdot V \delta t
\end{aligned}
$$

It can be noted that the above equation is identical to the momentum equation of continuum "phase" (5) with the source function being given by (24).

The unsteady equations (11),(19), and (23) for continuum density and momentum fields will be referred to as Generalised Landau-Lifshitz Fluctuating Hydrodynamics (GLL-FH) equations. These equations are to be solved together with the modified MD equations (14) and (15). Properties of the GLL-FH equations in some special limiting cases are analysed in the next section. 


\subsection{Special cases and the compatibility condition at the hydrodynamic boundary between the}

\section{pure continuum and the particle region}

1. If $s=0$ (pure MD case) then the density GLL-FH equation (19) reduces to:

$$
\frac{\delta_{t} \rho^{*}}{\delta t}=Q_{\rho}=-\frac{1}{V} \sum_{\gamma=1}^{6}\left[\left(\sum_{p=1}^{N_{\gamma}} u_{i p} \rho_{p}\right) d n_{i}^{\gamma}\right]
$$

and the momentum equation (23) becomes:

$$
\frac{\delta_{t} q_{i}}{\delta t}=Q_{i}=\sum_{p=1}^{N} F_{i p}{ }^{M D}-\frac{1}{V} \sum_{\gamma=1}^{6}\left[\sum_{p=1}^{N_{\gamma}}\left(u_{j p} \rho_{p} u_{i p}\right) d n_{j}^{\gamma}\right]
$$

From Eq.(11), abridged equations for the difference between the continuum and the MD fields are obtained:

$$
\frac{D \rho^{\prime}}{D t}=\frac{\delta_{t} \rho^{\prime}}{\delta t}+\frac{1}{V} \sum_{\gamma=1}^{6}\left(\rho^{\prime} \tilde{\mathbf{u}} \cdot d \mathbf{n}^{\gamma}\right)=0 \text { for mass }
$$

and

$$
\frac{D q_{i}^{\prime}}{D t}=\frac{\delta_{t} q_{i}^{\prime}}{\delta t}+\frac{1}{V} \sum_{\gamma=1}^{6}\left(q_{i}^{\prime} \tilde{\mathbf{u}} \cdot d \mathbf{n}^{\gamma}\right)=0 \quad \text { for momentum }
$$

Since the above equations are homogeneous, if the initial and boundary conditions are such that $\rho^{\prime}$ and $q_{i}^{\prime}$ are both equal to zero, these quantities will remain zero at all later times, hence,

$$
\tilde{\rho}=\rho^{*}, \quad \tilde{u}_{i}=q_{i} / \tilde{\rho}
$$

In this case, the solution fields obtained from MD particles, which are averaged over any control volume and time step of the continuum hydrodynamics representation, satisfy equations (19) and (23) exactly. That is, in the limit of $s \rightarrow 0$ the GLL-FH equations reduce to all-atom Molecular Dynamics not only in the sense of a sufficiently large statistical ensemble, as it is the case for the classical LandauLifshitz Fluctuating Dynamics equations (6)-(9), but also in terms of the locally averaged fields. 
2. $s=1$ corresponds to a pure hydrodynamic zone. In particular, this includes the vicinity of where the continuum hydrodynamics zone merges with the hybrid continuum-particle zone $(s<1, s \rightarrow 1)$. This is where an appropriate (e.g. periodic) boundary condition needs to be used for the MD particles when solving equations (14) and (15). Altogether, this sets an internal compatibility condition that must be satisfied between the MD particle and the continuum hydrodynamic regions for consistency as discussed below:

In the considered case of the particle-continuum zone, $s<1, s \rightarrow 1$, GLL-FH equations (19) and (23) reduce to

$$
\frac{D \rho^{*}}{D t}=\frac{\delta_{t} \rho^{*}}{\delta t}+\frac{1}{V} \sum_{\gamma=1}^{6}\left(\rho^{*} \tilde{\mathbf{u}} \cdot d \mathbf{n}^{\gamma}\right)=\alpha f(s) \rho^{\prime}=\alpha \cdot \alpha_{0} \rho^{\prime}
$$

and

$$
\frac{D q_{i}}{D t}=\frac{\delta_{t} q_{i}}{\delta t}+\frac{1}{V} \sum_{\gamma=1}^{6}\left(q_{i} \tilde{\mathbf{u}} \cdot d \mathbf{n}^{\gamma}\right)=F_{i}+\beta \cdot \alpha_{0} q_{i}^{\prime}
$$

In a similar way, equations (11) which describe the difference between the continuum and the particle phase density and momentum reduce to:

$$
\frac{D \rho^{\prime}}{D t}=\frac{\delta_{t} \rho^{\prime}}{\delta t}+\frac{1}{V} \sum_{\gamma=1}^{6}\left(\rho^{\prime} \tilde{\mathbf{u}} \cdot d \mathbf{n}^{\gamma}\right)=-\alpha \cdot \alpha_{0} \rho^{\prime}
$$

and

$$
\frac{D q_{i}^{\prime}}{D t}=\frac{\delta_{t} q_{i}^{\prime}}{\delta t}+\frac{1}{V} \sum_{\gamma=1}^{6}\left(q_{i}^{\prime} \tilde{\mathbf{u}} \cdot d \mathbf{n}^{\gamma}\right)=-\beta \cdot \alpha_{0} q_{i}^{\prime}
$$

The above equations lead to an exponential convergence of the $\rho^{\prime}$ and $q_{i}^{\prime}$ quantities to zero, hence, leading to $\tilde{\rho}=\rho^{*}, \tilde{u}_{i}=q_{i} / \tilde{\rho}$, and the $\rho^{\prime}$ and $q_{i}^{\prime}$ variables are completely decoupled from the LL-FH equations in the continuum hydrodynamic zone. 
From this, the classical continuum density and momentum LL-FH equations are satisfied for the mixture variables in the pure hydrodynamic region $(s=1)$ :

$$
\frac{D \tilde{\rho}}{D t}=\frac{\delta_{t} \tilde{\rho}}{\delta t}+\frac{1}{V} \sum_{\gamma=1}^{6}\left(\tilde{\rho} \tilde{\mathbf{u}} \cdot d \mathbf{n}^{\gamma}\right)=0
$$

and

$$
\frac{D}{D t}\left(\tilde{u}_{i} \tilde{\rho}\right)=\frac{\delta_{t}\left(\tilde{u}_{i} \tilde{\rho}\right)}{\delta t}+\frac{1}{V} \sum_{\gamma=1}^{6}\left(\tilde{u}_{i} \tilde{\rho} \tilde{\mathbf{u}} \cdot d \mathbf{n}^{\gamma}\right)=F_{i}
$$

Equations (27) and (28) play the role of compatibility conditions between the particle-continuum zone and the pure hydrodynamic zone. For continuity of mass and momentum fluxes, the same mass and momentum flux must be preserved across the interface $s=1$ that separates the pure hydrodynamic zone from the particle-continuum zone. Hence, instead of solving the reduced continuum equations (29) and (30) in the pure hydrodynamic zone, the equations are rearranged to the form of GLL-FH equations (19) and (23) for the continuum density and momentum fields $\rho^{*}=\tilde{\rho}-\rho^{\prime}$ and $q_{i}=\tilde{u}_{i} \tilde{\rho}-q_{i}^{\prime}$ . This results in a numerically consistent formulation where the same unified system of equations (25)-(28) is solved in the entire computational domain (fig.1).

\section{Computational implementation}

Following the computational method for solving the standard LL-FH equations ${ }^{37}$, a finite-volume scheme is implemented for the GLL-FH equations, which is formulated in a space-time staggered predictor-corrector form.

At the predictor step, the solution at the mid-time-level is updated using a forward time integration and a central flux approximation. This is followed by the second-order extrapolation step to compute the conservation fluxes at the new time level. The final step is corrector where the solution at the new time 
step is computed using a backward time integration. This results in a computational scheme that is second order in space and time. Further details of the algorithm are outlined in the Appendix.

A special note is in order to describe the "phase binding" parameters of the hybrid model, $\alpha$ and $\beta$ which have units of $\left[\mathrm{s}^{-1}\right]$. These parameters can be considered as some effective relaxation constants which prevent the continuum and the particle 'phases' from separation. The values of $\alpha$ and $\beta$ need to be sufficiently large in comparison with the hydrodynamic gradients for effective phase binding. In the numerical examples of the paper, $\alpha$ and $\beta$ are order of $1-10\left[\mathrm{ps}^{-1}\right]$ that corresponds to the characteristic time scale of 10-100 MD time steps. This time scale should be much smaller than a characteristic time of the hydrodynamic interaction. It can be noted that the range of $\alpha$ and $\beta$ parameters of the suggested hybrid multiscale method determines the applicability range of the method it terms of the assumed space-time scale separation between the small atomistic particle and the large continuum hydrodynamic scales.

In comparison with the GLL-FH equations, which are solved in the entire computational domain, the MD equations are solved in a small part of the domain. The computational cost of the best MD solvers, such as those implemented in GROMACS ${ }^{42-44}$, scales linearly with $N$ in ideal cases, where $N$ is the number of MD particles. For statistical averaging accuracy, the number of particles per control volume should be at least $\mathrm{O}(100)$ compared to the number of GLL-FH control volumes.

Since the same unified set of GLL-FH equations can be solved in the entire computational domain consistently without keeping a large number of control volumes occupied by the particles, the computational benefits of the suggested approach over the all-atom MD simulations can be significant. These benefits are illustrated in Table 1, which compares computational costs of the current hybrid method with the cost of all-atom MD simulations for the numerical examples considered in section 4. All hybrid model simulations considered (10 ns or $10^{6}$ MD time steps for liquid argon) typically take about 1 hour on a computer workstation equipped with Graphics Processing Units (GPUs). In 
comparison with this, the all-atom MD simulations for the same problems require a 4-6 times longer run time. Notably, the running times compared correspond to the same computer and the same GROMACS version, with and without including the GLL-FH model.

It can be further noted that efficiency of the new hybrid scheme is directly linked to the size of the continuum hydrodynamics zone relative to the total computational domain size. For macroscopic-size computational domains, the computational benefits of the new approach over the all-atom molecular dynamics simulations of the same domain size are expected to be many orders of magnitude larger in comparison with the current test cases.

Table 1: Simulation costs of the hybrid multiscale method against the all-atom molecular dynamics for typical test problems, hours per nanosecond $[\mathrm{hrs} / \mathrm{ns}]$ in each case

\begin{tabular}{|l|c|c|c|}
\hline \multicolumn{1}{|c|}{ Test case } & Steady equilibrium & Couette flow & $\begin{array}{c}\text { Acoustic wave } \\
\text { propagation }\end{array}$ \\
\hline $\begin{array}{l}\text { Cost of the all-atom } \\
\text { simulation }[\mathrm{hrs} / \mathrm{ns}] / \text { cost of } \\
\text { the hybrid method }[\mathrm{hrs} / \mathrm{ns}]\end{array}$ & $0.58 / 0.10$ & $0.58 / 0.10$ & $0.42 / 0.10$ \\
\hline
\end{tabular}

\section{Numerical Examples}

\subsection{Liquid argon at steady equilibrium conditions}

As the first test, the GLL-FH method solutions will be analysed for how well the particle/continuum "phases" bind together and preserve standard deviation of density and velocity of liquid argon fluctuations at thermal equilibrium and zero flow conditions. 
Following Ref. ${ }^{31}$, a cubic computational domain is considered first. At the open boundaries, periodic boundary conditions are applied. A spherical-shape user-defined function $s=s(\mathbf{x}, t)$ of the hybrid multiscale model based on the two-phase analogy is specified so that it is zero in the centre of the computational domain and grows to the domain periphery where the particles are driven by the external hydrodynamic field:

$$
s(r)= \begin{cases}S_{\min }, & r \leq R_{M D} ; \\ \frac{r-R_{M D}}{R_{F H}-R_{M D}}\left(S_{\max }-S_{\min }\right)+S_{\min }, & R_{M D}<r<R_{F H} ; \\ S_{\max }, & r \geq R_{F H} .\end{cases}
$$

where $R_{M D}$ and $R_{F H}$ are the radii of the discrete particle and the hydrodynamic zone which are userdefined parameters, $r$ is the distance to the geometrical center of discrete particle zone, $S_{\min }=0$ and $S_{\max }=1$ (fig. 1).

The density and velocity components of the GLL-FH solution are sampled in several points of the computational domain, which correspond to different values of the $s$-function, for subsequent analysis. For $s=0$ and $s=1$, which correspond to the centre and the periphery of the domain, respectively, the mixture velocity and density are equal to the corresponding MD velocity and density in the given cell within the statistical noise error. In the intermediate hybrid zone $(0<s<1)$, MD and mixture variables are driven to each other very fast (exponentially), as shown in Figures 2-3. For the same hybrid zone region, Figure 4 shows that the corresponding standard deviations of the density and velocity fluctuations are also in excellent agreement with the all-atom MD simulations within a statistical error. 


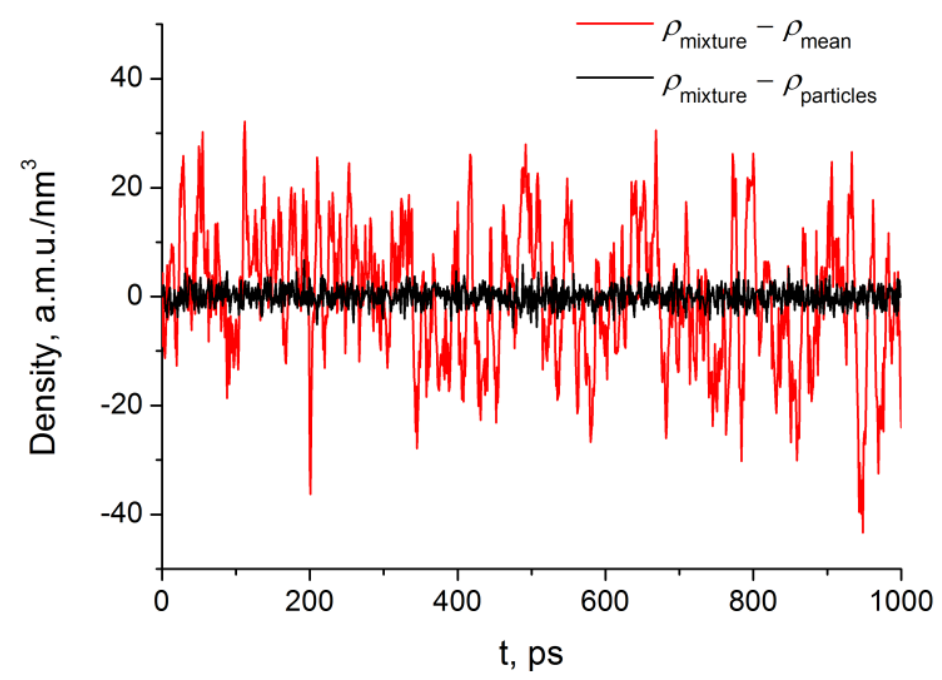

Figure 2. Time-history of density fluctuations of mixture and MD "phase", $s=0.5$



Figure 3. Time-history of velocity fluctuations of mixture and MD "phase", $s=0.5$ 

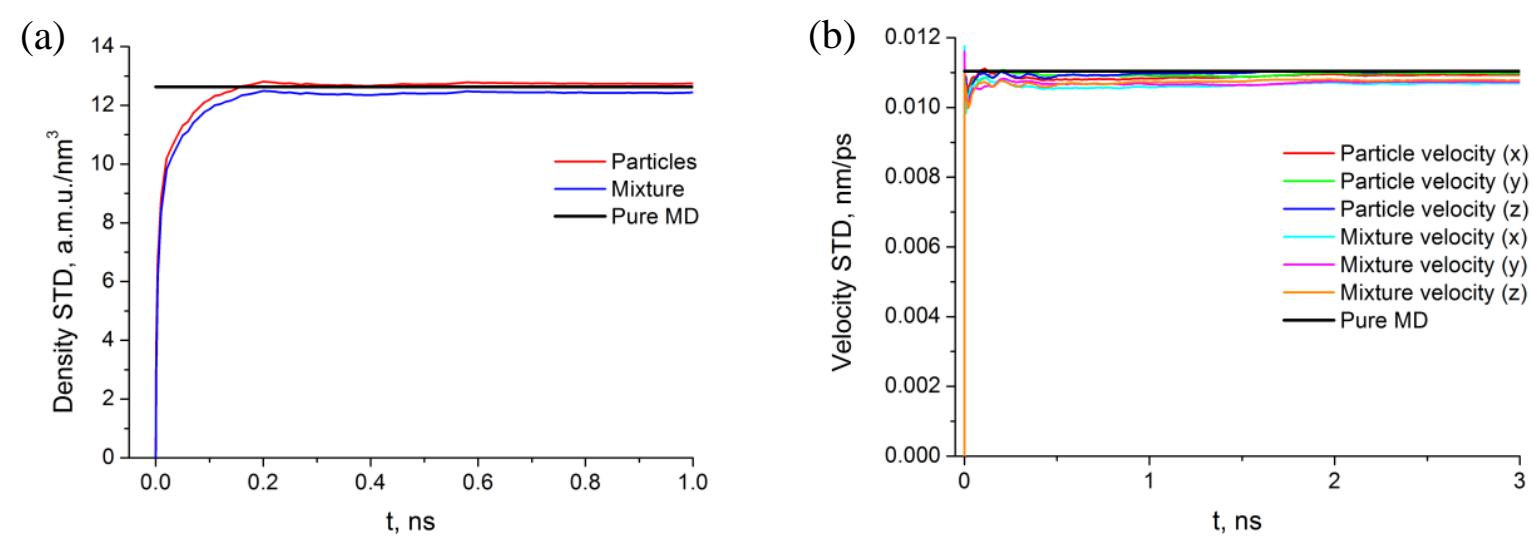

Figure 4. Standard deviations of density (a) and velocity (b) for $s=0.5$

Fig. 5 shows the comparison of the radial distribution and velocity autocorrelation of atoms, RDF and VACF, obtained in the pure MD region of the hybrid computational domain $(s=0)$ with the all-atom MD simulation. RDF and VACF are the standard measures of how well the microscopic structure is resolved within the molecular dynamics part of a multiscale model in terms of correctly capturing the microscopic probability distribution of atoms and their decorrelation time.

For the VACF distribution, the previous solution from the one-way coupled implementation of the hybrid multiscale method ${ }^{31}$ is compared with the current two-way coupled solution on the same plot.

In comparison with the current simulation, the previous one-way coupled hybrid model ignores the effect of atomistic scales on the continuum "phase" by approximating the mixture solution variables with the standard Landau-Lifshitz Fluctuating Hydrodynamics model everywhere in the computational domain. From the comparison, it is clear that the feedback effect from the molecular dynamics on the macroscopic field is important to take into account if the velocity autocorrelation function of atoms needs to be simulated accurately. 
(a)

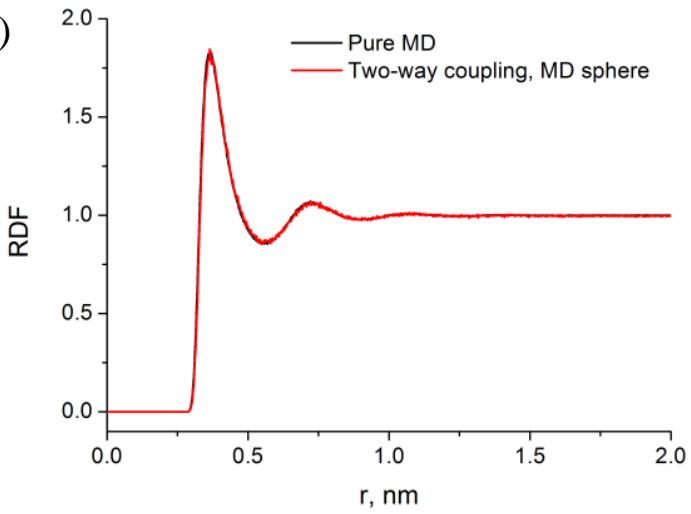

(b)

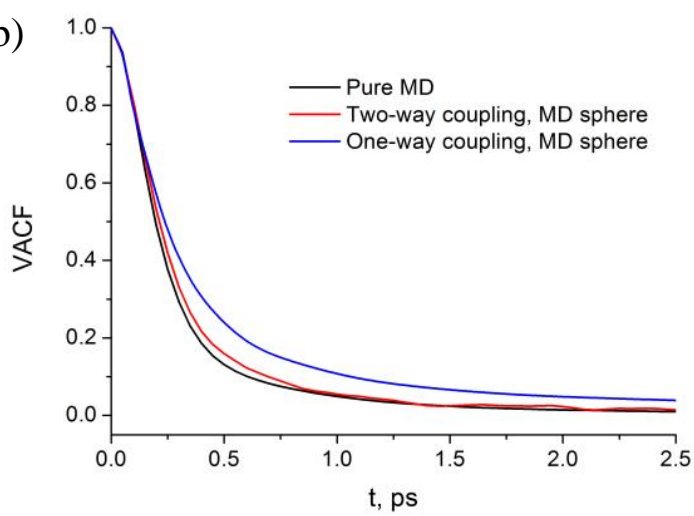

Figure 5. RDF (a) and VACF (b) in the MD zone, argon.

\subsection{Steady Couette flow}

The same cubic hybrid computational domain is considered by defining the spherically symmetric distribution of the user-defined function $s=s(\mathbf{x}, t)(31)$ to separate the zones of molecular dynamics and continuum fluid dynamics, which is now applied for the steady Couette flow test.

It can be noted that the steady Couette flow is an important feature of many engineering microfluidic applications, which could benefit from multi-resolution, continuum-atomistic modelling, such as the deformation of biomolecules by hydrodynamic gradients, which can occur in dual laminar flow microfluidic systems. ${ }^{45-47}$ While the unsteady start-up Couette flow problem will not be considered in the present paper, the capability of the suggested multiscale method for capturing unsteady flows will be tested in the acoustic wave propagation problem in section 4.3 .

Couette flow in liquid argon is implemented by incorporating an additional forcing term in the FH/hybrid region:

$$
\frac{\partial q_{i}}{\partial t}+\frac{\partial}{\partial x_{j}}\left(s \tilde{u}_{j} q_{i}\right)=Q_{i}+s F_{i}+\beta f(s) \cdot q_{i}^{\prime}+F_{i}^{\text {flow }},
$$

where 


$$
F_{x}^{\text {flow }}=-\varepsilon_{0} s\left(\left\langle q_{x}\right\rangle_{x, z} / \rho_{0}-u_{\text {analytical }}(y)\right), \quad F_{y}^{\text {flow }}=F_{z}^{\text {flow }}=0
$$

so there is a flow along the $x$ axis with the mean velocity which only depends on the $y$ coordinate, while the flow in the periodic $z$-direction is homogeneous (figure 6). The "target" analytical solution corresponds to the steady shear flow that depends just on the vertical coordinate in this case but generally could be a function of other space coordinates and time as well so long as its variation is slower than 10-100MD time steps (see discussion in Section 3).

It should be pointed out that the forcing term $F_{i}^{\text {flow }}$ is identically zero in the pure MD region, hence, the atoms in MD region are accelerated by the flow through their interaction with the particlehydrodynamic region at the periphery. That is, there is no artificial forcing applied to the atoms in the pure MD domain, which is simulated in a full accordance with the inter-atomic potentials of the standard equilibrium molecular dynamics. This can be compared with some of the Non-Equilibrium MD methods which apply an artificial force on all atoms directly to simulate the flow effect. ${ }^{48-50}$

Fig.7 compares the continuum velocity and the sub-cell averaged MD particle velocity distributions with the analytical solution for the Couette problem. Apparently, the "binding" of MD particles to the flow via the hybrid region at the periphery is sufficient to obtain an excellent agreement with the analytical solution. 


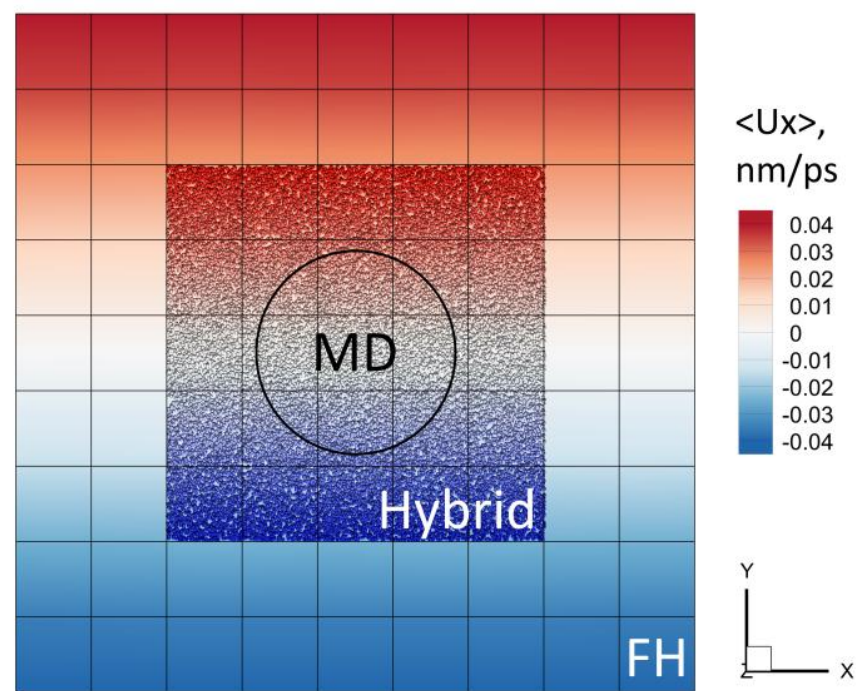

Figure 6. Couette flow in MD/FH region

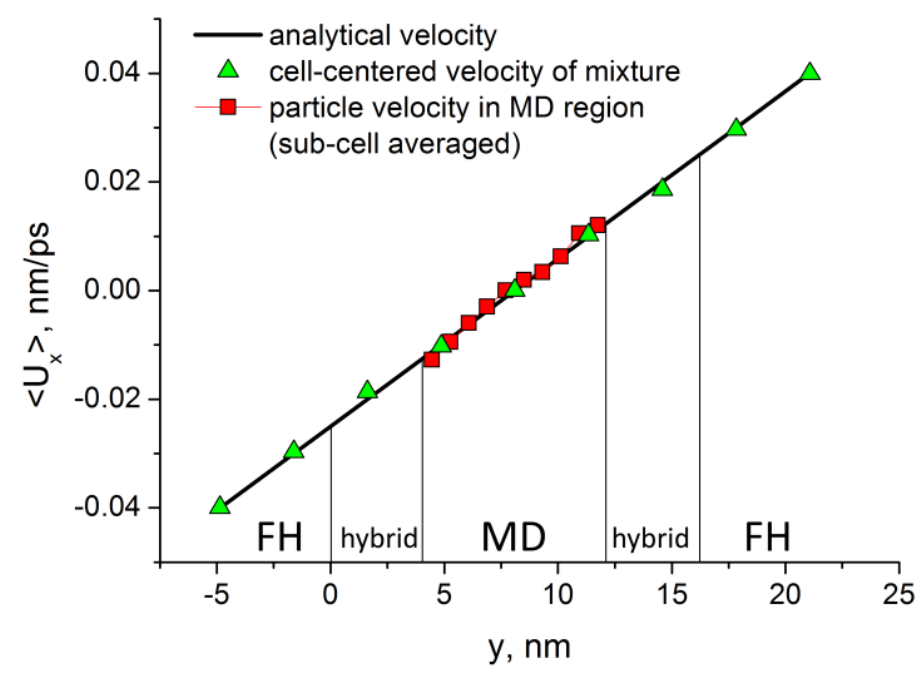

Figure 7. Couette flow profile for mixture (tilde) velocity and for particles' velocity in the MD region

Fig.8 shows the corresponding RDF and VACF distributions in the pure MD region with Couette flow. Similar to the stationary case, these distributions are in an excellent agreement with the reference (noflow) all-atom MD simulation. This indicates that, for the considered shear flow regime, the internal 
material structure of liquid argon is not affected by the shear flow. The latter property is common for Newtonian fluids and is similar to preservation of the molecular diffusion coefficient - another material characteristic of a gas or a liquid, which for Newtonian fluids does not depend on the velocity strain field in accordance with the Curie's theorem of linear irreversible thermodynamics. ${ }^{48}$

(a)

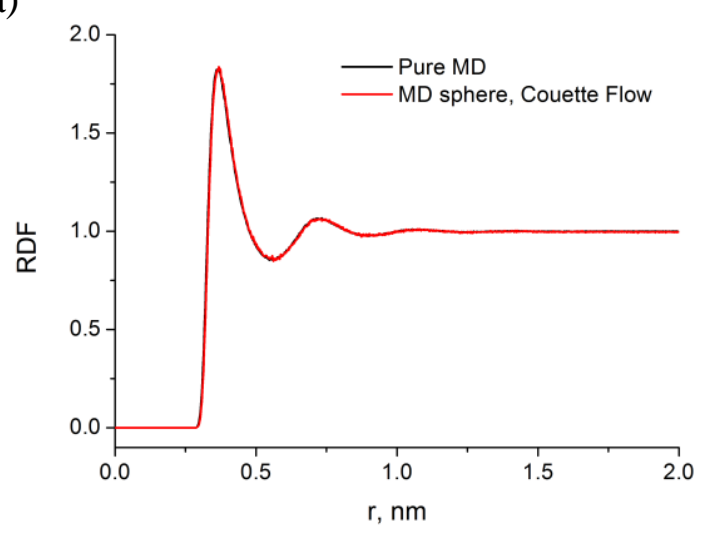

(b)



Figure 8. RDF (a) and VACF (b) in the MD zone with Couette flow

\subsection{Acoustic wave propagation}

As a final example, accuracy of the new AdResS-FH model is tested for an acoustic wave propagation problem following. ${ }^{25,51,52}$ It can be noted that in comparison with the shear wave propagation test problems considered by Ref. ${ }^{31}$ and Ref. ${ }^{53}$, acoustic wave propagation involves closely correlated fluctuations of density and velocity in accordance with the linear wave dispersion relation, hence, is more challenging for numerical modelling.

An elongated rectangular hybrid computational domain is considered with defining a one-dimensional distribution of the user-defined function $s=s(\mathbf{x}, t)$, which delineates the zones of molecular dynamics and continuum fluid dynamics for the acoustic wave propagation in the $x$-direction in a similar way to the spherical-shape $s$-function (31) (fig.9). 
An incoming planar acoustic wave solution is imposed at the inlet boundary of the hydrodynamic domain. Because of the central stencil of the scheme (see Appendix), the numerical implementation of the GLL-FH equations requires specifying the acoustic wave solution at first two cell layers in the pure hydrodynamic region for density and momentum variables, $\rho^{*}=\rho^{*}+\hat{\rho}, q_{i}=q_{i}+\hat{q}_{i}$, where

$$
\begin{gathered}
\hat{\rho}^{*}=\varepsilon\left\langle\rho^{*}\right\rangle \cos (\omega t-k x), \\
\hat{q}_{x}=\varepsilon c_{S}\left\langle\rho^{*}\right\rangle \cos (\omega t-k x) .
\end{gathered}
$$

Here $\omega=\frac{2 \pi c_{S}}{L_{x}}, k=\frac{2 \pi}{L_{x}}, L_{x}$ is the length of the box along $x$ axis, $c_{S}$ is speed of sound, which is determined in accordance with the equation of state of liquid argon.

The numerical resolution of solving the GLL-FH equations corresponds to 20 cells per acoustic wavelength, which resolution is considered adequate for the present second-order numerical scheme.

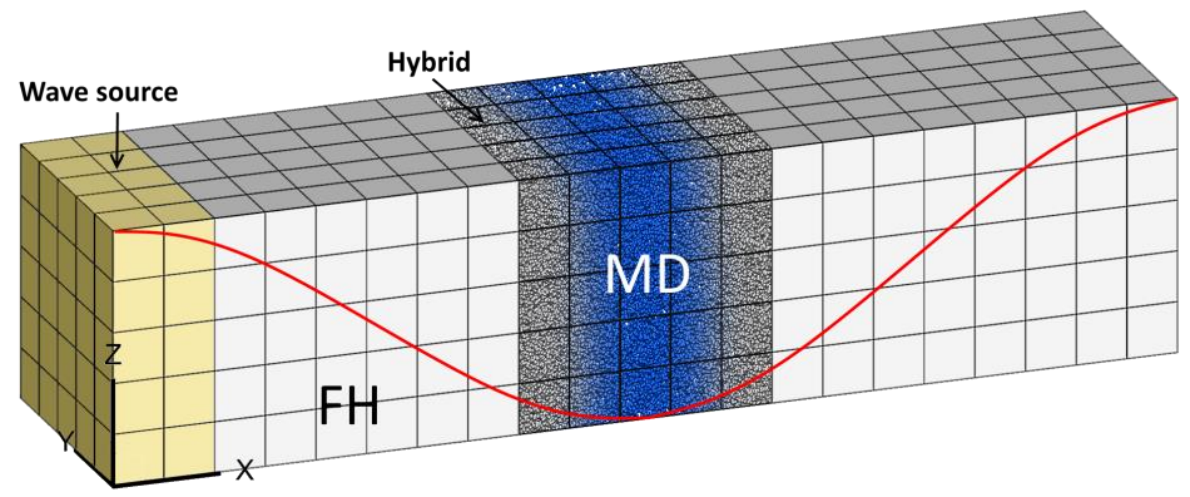

Figure 9. 3D view of the computational domain for the acoustic wave propagation problem $-21 \times 5 \times 5$ cells in total including the FH, hybrid, and MD region. The contour line shows a typical instantaneous acoustic wave distribution in argon which wavelength is equal to the computational domain size. 
Fig.10 shows several realisations of density and velocity signals extracted from the centre of the pure MD region. The total time window of the data extracted corresponds to $100 \mathrm{~ns}$ which is about 1000 acoustic time periods. Acoustic fluctuations are an order of magnitude smaller compared to the thermal fluctuations but compared to the former the latter are uncorrelated. Hence, to reduce the noise, all signals have been phase-averaged and also averaged in the $y-z$ plane which corresponds to the homogeneous directions of the problem.

The averaged MD density and velocity fields agree with the analytical solution within the statistical noise scatter. This indicates that the two-way coupled hybrid model correctly transports both the mass and momentum of the macroscopic fluctuations in the system.

(a)

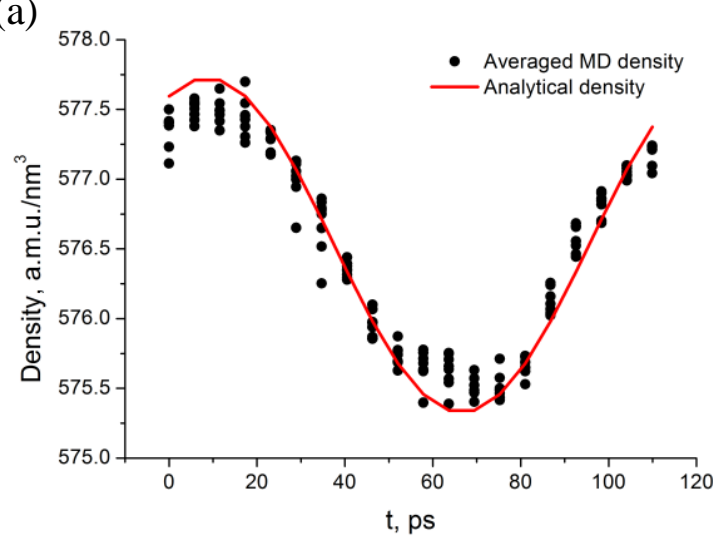

(b)

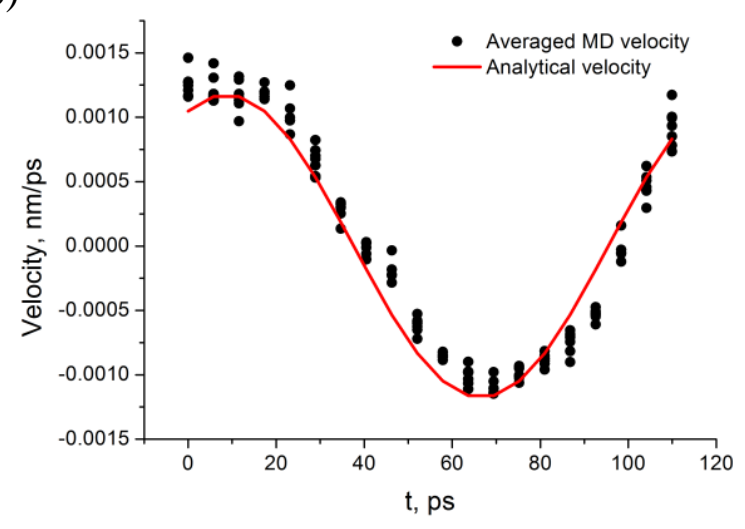

Figure 10. Mixture density (a) and velocity (b) averaged over acoustic wave phase in comparison with analytical solution after $100 \mathrm{~ns}(\sim 1000$ periods of acoustic wave)

It should be noted that in order to avoid spurious reflections of the acoustic wave from the particle/continuum boundaries, the model calibration parameters, e.g. $\alpha, \beta$, and $S_{\max }$ parameter of the $s$-function, had to be fine-tuned in the hybrid zone $0<s<1$. For this particular test, $\alpha$ and $\beta$ were increased to the maximum values as discussed in Section 3 to provide the strongest coupling between 
the particle and continuum "phases", while $S_{\max }$ in (31) for the hybrid region was slightly reduced to achieve a smooth gradient of the $s$-function in the particle zone, hence, a smooth solution behaviour near the discrete particle/continuum boundary.

It can be further remarked that in comparison with some previous works of the authors, e.g. Refs. ${ }^{31,36}$, where the analytical acoustic solution was directly imposed as a part of the one-way coupled approach, the statistical noise level of the two-way coupled solution is expected to be higher because of the nonlinearity and stochastic nature of the GLL-FH equations as one approaches the MD resolution scale.

\section{Conclusion}

A new Generalised Landau-Lifshitz Fluctuating Hydrodynamic (GLL-FH) model is proposed for consistent two-way coupling between the molecular dynamics and continuum hydrodynamics of the same fluid in the framework of analogy with two-phase hydrodynamics. The new model is based on extending the classical Landau-Lifshitz fluctuating hydrodynamic equations in order to record and propagate the differences between the locally averaged atomistic particle fields and the continuum hydrodynamic fields in accordance with the conservation laws. In comparison with the previous models, including the work of the authors, the new model allows one to consistently take the feedback from the particle dynamics on the continuum fluid dynamics into account concurrently. This feedback is important for tailoring the macroscopic fluid dynamics equations to the dynamics of discrete particle dynamics on-the-fly rather than prescribing the former from an approximate analytical model such as the standard Landau-Lifshitz Fluctuating Hydrodynamics model that is restricted to the Gaussian statistics. In terms of numerical implementation, the inclusion of the feedback effect results in a less stiff coupling between the continuum and discrete particle parts of the hybrid model in comparison with the previous one-way coupled models. Numerical examples are considered for several simulations of liquid argon flows in GROMACS, a popular open-source molecular dynamics software. The 
improved consistency of the GLL-FH model has facilitated the current implementation of an efficient spatial domain decomposition of the zone of discrete particles from the pure continuum hydrodynamic zone in a seamless way. Such spatial decomposition was not possible to implement in the previous hybrid multiscale models based on the two-phase analogy, which lacked the full consistency. Thanks to the new implementation, an order of magnitude reduction in computational cost has been achieved for the current hybrid model implementation in comparison with the all-atom molecular dynamics solution. An excellent agreement of the solutions of the new two-way coupled hybrid method with the reference all-atom molecular dynamics simulations is demonstrated for the benchmark problem of liquid argon fluctuations in equilibrium. The solutions for the velocity autocorrelation function of atoms obtained with the new method show a superior accuracy in comparison with the previous oneway coupled hybrid multiscale method that ignored the feedback effect on the flow. As a demonstration of new capabilities of the two-phase flow analogy method to cope with non-uniform macroscopic flows, both the macroscopic velocity and the microscopic structure properties of liquid argon under steady Couette flow conditions are obtained from the numerical simulation. The numerical solutions are in a good agreement with the analytical solution for the meanflow velocity profile and the reference all-atom molecular dynamics simulation in the absence of flow. The latter is in accordance with the physics of Newtonian fluids, such as argon, whose intrinsic material properties do not depend on the flow applied. As a final example, the new GLL-FH model is tested on the problem of high-frequency acoustic wave propagation in liquid argon. After the normal-plane and phase averaging applied, the solution of the GLL-FH equations demonstrates a good agreement with the reference analytical solution both in terms of the amplitude and the phase.

As a final remark, it should be emphasised that the suggested GLL-FH method provides a general unified framework for coupling MD particles and continuum fluid dynamics. In particular, in all current examples, a simple monoatomic liquid is considered, which is represented by a single (allatom) resolution MD model as the particle "phase" of the GLL-FH equations. However, the suggested 
GLL-FH modelling framework can also be applied to multi-resolution particle (e.g. AdResS) simulations for modelling of complex liquids, e.g. protein solutions in water, which may require additional measures for the reduction of complexity of a discrete particle system before coupling the latter with continuum hydrodynamics. The required complexity reduction can be achieved, for example, by adopting the recent work ${ }^{36}$ of the authors who implemented AdResS in the framework of the two-phase analogy method.

\section{Acknowledgments}

This project has received funding from the European Union's Horizon 2020 research and innovation programme under the Marie Sklodowska-Curie grant agreement No 700276 (Individual Fellowship Grant H2020-MSCA-IF-2015-700276 HIPPOGRIFFE).

\section{Appendix: Numerical Implementation}

For solving the MD particle equations (14) and (15), the following standard leap-frog algorithm is used to advance the coordinates and velocities over one MD time step:

For atom coordinates:

$$
\frac{x_{i p}\left(t+\Delta t_{M D}\right)-x_{i p}(t)}{\Delta t_{M D}}=\left(1-s_{p}\right) u_{i p}\left(t+\frac{1}{2} \Delta t_{M D}\right)+s_{p} \tilde{u}_{i p}^{n+1 / 2}-\alpha \int_{x_{0 i}}^{x_{i}}\left(f(s) \rho^{\prime n+1 / 2} / \sum_{p=1, N} \rho_{p}(t)\right) d y_{i},
$$


For atom velocities:

$$
\begin{aligned}
& \frac{u_{i p}\left(t+\frac{1}{2} \Delta t_{M D}\right)-u_{i p}\left(t-\frac{1}{2} \Delta t_{M D}\right)}{\Delta t_{M D}}=\left(1-s_{p}\right) F_{i p}^{M D}(t) / \rho_{p}(t)+s_{p} F_{i p}^{n+1 / 2} / \sum_{p=1}^{N} \rho_{p}(t)- \\
& -\left[\frac{1}{V} \sum_{\gamma=1}^{6}\left(\sum_{p=1, N} \alpha u_{i p}(t) \rho_{p}(t)\left[\int_{x_{0 j}}^{x_{j}}\left(f(s) \rho^{\prime n+1 / 2} / \sum_{p=1, N} \rho_{p}(t)\right) d y_{j}\right]\right) d n_{j}^{\gamma}\right]_{p} / \sum_{p=1, N} \rho_{p}(t)+ \\
& +\beta\left[f(s) q_{i}^{\prime n+1 / 2}\right]_{p} / \sum_{p=1}^{N} \rho_{p}(t)
\end{aligned}
$$

Here superscript $n$ denotes the time layer index in the FH model, for example, $\rho^{\prime n} \equiv \rho^{\prime}(t)$, $\rho^{\prime n+1 / 2} \equiv \rho^{\prime}\left(t+\frac{1}{2} \Delta t_{F H}\right), \rho^{\prime n+1} \equiv \rho^{\prime}\left(t+\Delta t_{F H}\right)$. Usually, $\Delta t_{F H} \gg \Delta t_{M D}$. Parameter $s$ without index $p$ is a mass-weighted value $s=\sum_{p=1}^{N}\left(s_{p} \rho_{p}\right) / \sum_{p=1}^{N} \rho_{p}$ (in case of variable $s$ ).

Integral $\int_{x_{0 i}}^{x_{i}}\left(f(s) \rho^{\prime n+1 / 2} / \sum_{p=1, N} \rho_{p}\right) d y_{i}$ is approximated in accordance with the $2^{\text {nd }}$ order trapezia formula

$$
\begin{aligned}
& \int_{x_{0 i}}^{x_{i}}\left(f(s) \rho^{\prime} / \sum_{p=1, N} \rho_{p}\right) d y_{i} \approx f(s) \rho_{\text {cell }(i)}^{\prime} /\left[\sum_{p=1, N} \rho_{p}\right]_{p \in \operatorname{cell}(i)}\left(\frac{h_{x, \operatorname{cell}(i)}}{2}\right)+ \\
& +f(s) \rho_{\text {cell }(i-1)}^{\prime} /\left[\sum_{p=1, N} \rho_{p}\right]_{p \in \operatorname{cell}(i-1)}\left(h_{x, \operatorname{cell}(i-1)}\right)+\ldots+f(s) \rho_{\operatorname{cell}\left(i_{0}\right)}^{\prime} /\left[\sum_{p=1, N} \rho_{p}\right]_{p \in \operatorname{cell}\left(i_{0}\right)}\left(h_{x, \operatorname{cell}\left(i_{0}\right)}\right)
\end{aligned},
$$

where $h_{x, \text { cell }(i)}$ is cell $i$ size along $x$ axis, $i_{0}$ is the index of the middle cell. In case $x_{i}<x_{0 i}$, the integral can be calculated the same way but with accounting for the opposite sign.

The continuum equations to be solved numerically are summarised below:

$$
\begin{gathered}
\frac{\delta_{t} \rho^{\prime}}{\delta t}+\frac{1}{V} \sum_{\gamma=1}^{6}\left(\rho^{\prime} \tilde{\mathbf{u}} \cdot d \mathbf{n}^{\gamma}\right)=-\alpha \cdot s(1-s)\left(\tilde{\rho}-\sum_{p=1}^{N} \rho_{p}\right)-\alpha \cdot \alpha_{0} s \rho^{\prime} \\
\frac{\delta_{t} q_{i}^{\prime}}{\delta t}+\frac{1}{V} \sum_{\gamma=1}^{6}\left(q_{i}^{\prime} \tilde{\mathbf{u}} \cdot d \mathbf{n}^{\gamma}\right)=-\beta s(1-s)\left(\tilde{\rho} \tilde{u}_{i}-\sum_{p=1}^{N} \rho_{p} u_{i p}\right)-\beta \cdot \alpha_{0} s q_{i}^{\prime}
\end{gathered}
$$




$$
\begin{gathered}
\frac{\delta_{t} \rho^{*}}{\delta t}+\frac{1}{V} \sum_{\gamma=1}^{6}\left[s \rho^{*} \tilde{\mathbf{u}} \cdot d \mathbf{n}^{\gamma}\right]=Q_{\rho}+\alpha f(s) \rho^{\prime}, \quad Q_{\rho}=-\frac{1}{V} \sum_{\gamma=1}^{6}\left[\left(\sum_{p=1}^{N_{\gamma}}\left(1-s_{p}\right) u_{i p} \rho_{p}\right) d n_{i}^{\gamma}\right] \\
\frac{\delta_{t} q_{i}}{\delta t}+\frac{1}{V} \sum_{\gamma=1}^{6}\left[s q_{i} \tilde{\mathbf{u}} \cdot d \mathbf{n}^{\gamma}\right]=Q_{i}+s F_{i}+\beta f(s) \cdot q_{i}^{\prime}, \\
Q_{i}=\sum_{p=1}^{N}\left[\left(1-s_{p}\right) F_{i p}{ }^{M D}\right]-\frac{1}{V} \sum_{\gamma=1}^{6}\left[\sum_{p=1}^{N_{\gamma}}\left(\left(1-s_{p}\right) u_{j p} \rho_{p} u_{i p}\right) d n_{j}^{\gamma}\right] \\
\tilde{\rho}=\rho^{*}+\rho^{\prime} \\
\tilde{u}_{i}=\left(q_{i}+q_{i}^{\prime}\right) / \tilde{\rho} .
\end{gathered}
$$

There are 12 equations (A4)-(A10) and 12 variables to solve: $\tilde{\rho}, \tilde{u}_{i}, \rho^{\prime}, q_{i}^{\prime}, \rho^{*}, q_{i}, i=x, y, z$.

Let us denote $\langle f(t)\rangle^{n}=\frac{1}{\Delta t_{F H}} \int_{t_{n}-\Delta t_{F H}}^{t_{n}} f(t) \cdot d t$ as a moving average of each relevant MD field over FH time step. For simplicity, details of the numerical algorithm will be demonstrated for the $x$ direction of $(x, y, z)$ Cartesian coordinate system.

Figure 11 shows the computational stencil in space in the one-dimensional case where subscripts C, CL, CR correspond to the cell centres, subscripts L, R, LL, RR refer to the cell sides.

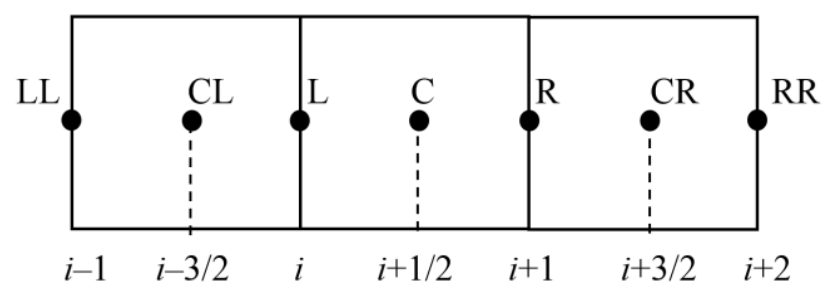

Figure 11. Spatial computational stencil for the $1 \mathrm{~d}$ scheme

Predictor step of the numerical algorithm for the continuum equations: 


$$
\begin{gathered}
\frac{\rho_{C}^{\prime n+1 / 2}-\rho_{C}^{\prime n}}{\Delta t_{F H} / 2}+\frac{\tilde{u}_{x, R}^{n}\left[\frac{1}{2}\left(\rho_{C R}^{\prime n}+\rho_{C}^{\prime n}\right)\right]-\tilde{u}_{x, L}^{n}\left[\frac{1}{2}\left(\rho_{C}^{\prime n}+\rho_{C L}^{\prime n}\right)\right]}{h_{x}}= \\
=-\alpha \cdot s_{C}\left(1-s_{C}\right)\left(\tilde{\rho}_{C}^{n}-\left\langle\sum_{p=1}^{N} \rho_{p}\right\rangle_{C}^{n}\right)-\alpha \cdot \alpha_{0} s_{C} \rho_{C}^{\prime n} \\
\frac{q_{i, C}^{\prime n+1 / 2}-q_{i, C}^{\prime n}}{\Delta t_{F H} / 2}+\frac{\tilde{u}_{x, R}^{n}\left[\frac{1}{2}\left(q_{i, C R}^{\prime n}+q_{i, C}^{\prime n}\right)\right]-\tilde{u}_{x, L}^{n}\left[\frac{1}{2}\left(q_{i, C}^{\prime n}+q_{i, C L}^{\prime n}\right)\right]}{h_{x}}= \\
=-\beta s_{C}\left(1-s_{C}\right)\left(\tilde{\rho}_{C}^{n} \tilde{u}_{i, C}^{n}-\left\langle\sum_{p=1}^{N} \rho_{p} u_{i p}\right\rangle_{C}^{n}\right)-\beta \cdot \alpha_{0} s_{C} q_{i, C}^{\prime n} \\
\frac{\rho_{C}^{* n+1 / 2}-\rho_{C}^{* n}}{\Delta t_{F H} / 2}+\frac{s_{R} \tilde{u}_{x, R}^{n}\left(\tilde{\rho}_{R}^{n}-\frac{1}{2}\left(\rho_{C R}^{\prime n}+\rho_{C}^{\prime n}\right)\right)-s_{L} \tilde{u}_{x, L}^{n}\left(\tilde{\rho}_{L}^{n}-\frac{1}{2}\left(\rho_{C}^{\prime n}+\rho_{C L}^{\prime n}\right)\right)}{h_{x}}=Q_{\rho, C}^{n}+\alpha f\left(s_{C}\right) \rho_{C}^{\prime n},
\end{gathered}
$$

where $Q_{\rho, C}^{n}$ is a counter of particle mass source/sink in cell $C$ and at time layer $n$ :

$$
\begin{gathered}
Q_{\rho}=-\frac{1}{V} \sum_{\gamma=1}^{6}\left[\left(\sum_{p=1}^{N_{\gamma}}\left(1-s_{p}\right) u_{i p} \rho_{p}\right) d n_{i}^{\gamma}\right] . \\
\frac{q_{i, C}^{n+1 / 2}-q_{i, C}^{n}}{\Delta t_{F H} / 2}+\frac{s_{R} \tilde{u}_{x, R}^{n}\left(\tilde{u}_{i, R}^{n} \tilde{\rho}_{R}^{n}-\frac{1}{2}\left(q_{i, C R}^{\prime n}+q_{i, C}^{\prime n}\right)\right)-s_{L} \tilde{u}_{x, L}^{n}\left(\tilde{u}_{i, L}^{n} \tilde{\rho}_{L}^{n}-\frac{1}{2}\left(q_{i, C}^{\prime n}+q_{i, C L}^{\prime n}\right)\right)}{h_{x}}= \\
=Q_{i, C}^{n}+s_{C} F_{i, C}^{n}+\beta f\left(s_{C}\right) \cdot q_{i, C}^{\prime n}
\end{gathered}
$$

where $Q_{i, C}^{n}$ is a counter of particle momentum source/sink in the given cell $C$ and time layer $n$ :

$$
\begin{gathered}
Q_{i}=\sum_{p=1}^{N}\left[\left(1-s_{p}\right) F_{i p}{ }^{M D}\right]-\frac{1}{V} \sum_{\gamma=1}^{6}\left[\sum_{p=1}^{N_{\gamma}}\left(\left(1-s_{p}\right) u_{j p} \rho_{p} u_{i p}\right) d n_{j}^{\gamma}\right] \\
\tilde{\rho}_{C}^{n+1 / 2}=\rho_{C}^{* n+1 / 2}+\rho_{C}^{\prime n+1 / 2} \\
\tilde{u}_{i, C}^{n+1 / 2}=\left(q_{i, C}^{n+1 / 2}+q_{i, C}^{\prime n+1 / 2}\right) / \tilde{\rho}_{C}^{n+1 / 2}
\end{gathered}
$$


The predictor step is followed by the extrapolation step, where the new flux variables $\tilde{\rho}^{n+1}$ and $\tilde{u}_{i}^{n+1}$ on the cell sides (faces) are determined using a linear extrapolation of the Riemann characteristic variable to compute the solution at the new time layer $n+1$. The characteristic decomposition procedure follows the work $^{37}$ which has 3 main steps:

1) The primary variables, $\rho$ and $u_{i}$, at the current time level $n$ and the intermediate time level $n+1 / 2$ are decomposed into the characteristic Riemann invariants, $R^{+}$and $R^{-}$in accordance with the hyperbolic part of the Navier-Stokes equations (6)-(9), which corresponds to isothermal gas dynamics equations: $R_{i}^{+}=u_{i}+c_{S} \log \left(\rho / \rho_{0}\right), R_{i}^{-}=u_{i}-c_{S} \log \left(\rho / \rho_{0}\right)$, where $\rho_{0}$ is a reference bulk fluid density and $c_{S}$ is the sound speed which is consistent with the fluid equation of state $p=p(\rho)$.

2) A weighted average of the second-order upwind and the second-order central extrapolation schemes with flux correction is applied to update the values of the invariants, $R^{+}$and $R^{-}$, at the new time level $n+1$ at the cell-face points by using the characteristic variables obtained at step 1 for the preceding time levels $n+1 / 2$ and $n$.

3) The final values of the flux variables $\rho^{n+1}$ and $u_{i}^{n+1}$ are obtained from the characteristic variables in accordance with their definition: $u_{i}=\left(R_{i}^{+}+R_{i}^{-}\right) / 2, \rho=\rho_{0} \exp \left(\frac{R^{+}-R^{-}}{2 c_{S}}\right)$.

\section{Corrector step:}

$$
\begin{aligned}
& \frac{\rho_{C}^{\prime n+1}-\rho_{C}^{\prime n+1 / 2}}{\Delta t_{F H} / 2}+ \\
& +\frac{\tilde{u}_{x, R}^{n+1}\left[\frac{1}{2}\left(2 \rho_{C R}^{\prime n+1 / 2}-\rho_{C R}^{\prime n}+2 \rho_{C}^{\prime n+1 / 2}-\rho_{C}^{\prime n}\right)\right]-\tilde{u}_{x, L}^{n+1}\left[\frac{1}{2}\left(2 \rho_{C}^{\prime n+1 / 2}-\rho_{C}^{\prime n}+2 \rho_{C L}^{\prime n+1 / 2}-\rho_{C L}^{\prime n}\right)\right]}{h_{x}}= \\
& =-\alpha \cdot s_{C}\left(1-s_{C}\right)\left(\tilde{\rho}_{C}^{n}-\left\langle\sum_{p=1}^{N} \rho_{p}\right\rangle_{C}^{n}\right)-\alpha \cdot \alpha_{0} s_{C} \rho_{C}^{\prime n}
\end{aligned}
$$




$$
\begin{aligned}
& \frac{q_{i, C}^{\prime n+1}-q_{i, C}^{\prime n+1 / 2}}{\Delta t_{F H} / 2}+ \\
& +\frac{\tilde{u}_{x, R}^{n+1}\left[\frac{1}{2}\left(2 q_{i, C R}^{\prime n+1 / 2}-q_{i, C R}^{\prime n}+2 q_{i, C}^{\prime n+1 / 2}-q_{i, C}^{\prime n}\right)\right]-\tilde{u}_{x, L}^{n+1}\left[\frac{1}{2}\left(2 q_{i, C}^{\prime n+1 / 2}-q_{i, C}^{\prime n}+2 q_{i, C L}^{\prime n+1 / 2}-q_{i, C L}^{\prime n}\right)\right]}{h_{x}}= \\
& =-\beta s_{C}\left(1-s_{C}\right)\left(\tilde{\rho}_{C}^{n} \tilde{u}_{i, C}^{n}-\left\langle\sum_{p=1}^{N} \rho_{p} u_{i p}\right\rangle_{C}^{n}\right)-\beta \cdot \alpha_{0} s_{C} q_{i, C}^{\prime n} \\
& \frac{\rho_{C}^{* n+1}-\rho_{C}^{* n+1 / 2}}{\Delta t_{F H} / 2}+\frac{s_{R} \tilde{u}_{x, R}^{n+1}\left(\tilde{\rho}_{R}^{n+1}-\frac{1}{2}\left(\rho_{C R}^{\prime n+1}+\rho_{C}^{\prime n+1}\right)\right)-s_{L} \tilde{u}_{x, L}^{n+1}\left(\tilde{\rho}_{L}^{n+1}-\frac{1}{2}\left(\rho_{C}^{\prime n+1}+\rho_{C L}^{\prime n+1}\right)\right)}{h_{x}}= \\
& =Q_{\rho, C}^{n+1}+\alpha f\left(s_{C}\right) \rho_{C}^{\prime n+1} \\
& \frac{q_{i, C}^{n+1}-q_{i, C}^{n+1 / 2}}{\Delta t_{F H} / 2}+\frac{s_{R} \tilde{u}_{x, R}^{n+1}\left(\tilde{u}_{i, R}^{n+1} \tilde{\rho}_{R}^{n+1}-\frac{1}{2}\left(q_{i, C R}^{\prime n+1}+q_{i, C}^{\prime n+1}\right)\right)-s_{L} \tilde{u}_{x, L}^{n+1}\left(\tilde{u}_{i, L}^{n+1} \tilde{\rho}_{L}^{n+1}-\frac{1}{2}\left(q_{i, C}^{\prime n+1}+q_{i, C L}^{\prime n+1}\right)\right)}{h_{x}}= \\
& =Q_{i, C}^{n+1}+s_{C} F_{i, C}^{n+1}+\beta f\left(s_{C}\right) \cdot q_{i, C}^{\prime n+1} \\
& \tilde{u}_{i, C}^{n+1}=\left(q_{i, C}^{n+1}+q_{i, C}^{\prime n+1}\right) / \tilde{\rho}_{C}^{n+1}
\end{aligned}
$$




\section{References}

1. Karabasov S, Nerukh D, Hoekstra A, Chopard B, Coveney P V. Multiscale modelling: approaches and challenges. Philos Trans $R$ Soc A Math Phys Eng Sci. 2014;372(2021):20130390-20130390. doi:10.1098/rsta.2013.0390

2. Gingold RA, Monaghan JJ. Smoothed particle hydrodynamics: theory and application to nonspherical stars. Mon Not R Astron Soc. 1977;181(3):375-389. doi:10.1093/mnras/181.3.375

3. Lucy LB. A numerical approach to the testing of the fission hypothesis. Astron J. 1977;82:1013. doi:10.1086/112164

4. Bhatnagar PL, Gross EP, Krook M. A model for collision processes in gases. I. Small amplitude processes in charged and neutral one-component systems. Phys Rev. 1954;94(3):511-525. doi:10.1103/PhysRev.94.511

5. Chen S, Doolen GD. Lattice Boltzmann Method For Fluid Flows. Annu Rev Fluid Mech. 1998;30(1):329-364. doi:10.1146/annurev.fluid.30.1.329

6. $\quad$ Pironneau O. Finite Element Methods for Fluids. John Wiley \& Sons; 1989.

7. Turner MJ, Clough RW, Martin HC, Topp LJ. Stiffness and Deflection Analysis of Complex Structures. J Aeronaut Sci. 1956;23(9):805-823. doi:10.2514/8.3664

8. Liu G-R, Quek SS. The Finite Element Method: A Practical Course. Butterworth-Heinemann; 2013.

9. Versteeg HK, Malalasekera W. An Introduction to Computational Fluid Dynamics: The Finite Volume Method. Pearson Education; 2007.

10. Karniadakis G, Beskok A, Aluru N. Microflows and Nanoflows: Fundamentals and Simulation (Interdisciplinary Applied Mathematics).; 2005. 
11. Patronis A, Lockerby DA. Multiscale simulation of non-isothermal microchannel gas flows. $J$ Comput Phys. 2014;270:532-543. doi:10.1016/j.jcp.2014.04.004

12. Ritos K, Borg MK, Lockerby DA, Emerson DR, Reese JM. Hybrid molecular-continuum simulations of water flow through carbon nanotube membranes of realistic thickness. Microfluid Nanofluidics. 2015;19(5):997-1010. doi:10.1007/s10404-015-1617-х

13. Teschner TR, Könözsy L, Jenkins KW. Progress in particle-based multiscale and hybrid methods for flow applications. Microfluid Nanofluidics. 2016;20(4):68. doi:10.1007/s10404016-1729-y

14. Español P, Warren PB. Perspective: Dissipative particle dynamics. J Chem Phys. 2017;146(15):150901. doi:10.1063/1.4979514

15. Delgado-Buscalioni R, Coveney P V. Continuum-particle hybrid coupling for mass, momentum, and energy transfers in unsteady fluid flow. Phys Rev E - Stat Physics, Plasmas, Fluids, Relat Interdiscip Top. 2003;67(4):13. doi:10.1103/PhysRevE.67.046704

16. Longshaw SM, Borg MK, Ramisetti SB, et al. mdFoam+: Advanced molecular dynamics in OpenFOAM. Comput Phys Commun. 2018;224:1-21. doi:10.1016/J.CPC.2017.09.029

17. Walther JH, Ritos K, Cruz-Chu ER, Megaridis CM, Koumoutsakos P. Barriers to Superfast Water Transport in Carbon Nanotube Membranes. Nano Lett. 2013;13(5):1910-1914. doi:10.1021/nl304000k

18. Holland DM, Lockerby DA, Borg MK, Nicholls WD, Reese JM. Molecular dynamics presimulations for nanoscale computational fluid dynamics. Microfluid Nanofluidics. 2015;18(3):461-474. doi:10.1007/s10404-014-1443-6

19. O'Connell ST, Thompson PA. Molecular dynamics-continuum hybrid computations: A tool for studying complex fluid flows. Phys Rev E. 1995;52(6):R5792-R5795. doi:10.1103/PhysRevE.52.R5792 
20. Hadjiconstantinou NG, Patera AT. Heterogeneous Atomistic-Continuum Representations for Dense Fluid Systems. Int J Mod Phys C. 1997;8(4):967-976. doi:10.1142/S0129183197000837

21. Nie XB, Chen SY, E WN, Robbins MO. A continuum and molecular dynamics hybrid method for micro- and nano-fluid flow. $J$ Fluid Mech. 2004;500:55-64. doi:10.1017/S0022112003007225

22. FlekkØy EG, Wagner G, Feder J. Hybrid model for combined particle and continuum dynamics. Europhys Lett. 2000;52(3):271-276. doi:10.1209/ep1/i2000-00434-8

23. Walther JH, Praprotnik M, Kotsalis EM, Koumoutsakos P. Multiscale simulation of water flow past a C540 fullerene. J Comput Phys. 2012;231(7):2677-2681. doi:10.1016/j.jcp.2011.12.015

24. Praprotnik M, Delle Site L, Kremer K. Adaptive resolution molecular-dynamics simulation: Changing the degrees of freedom on the fly. J Chem Phys. 2005;123(22):224106. doi:10.1063/1.2132286

25. Delgado-Buscalioni R, Kremer K, Praprotnik M. Concurrent triple-scale simulation of molecular liquids. J Chem Phys. 2008;128(11):114110. doi:10.1063/1.2890729

26. Markesteijn A, Karabasov S, Scukins A, Nerukh D, Glotov V, Goloviznin V. Concurrent multiscale modelling of atomistic and hydrodynamic processes in liquids. Philos Trans $R$ Soc A Math Phys Eng Sci. 2014;372(2021):20130379-20130379. doi:10.1098/rsta.2013.0379

27. Pavlov E, Taiji M, Scukins A, Markesteijn A, Karabasov S, Nerukh D. Visualising and controlling the flow in biomolecular systems at and between multiple scales: From atoms to hydrodynamics at different locations in time and space. Faraday Discuss. 2014;169:285-302. doi:10.1039/c3fd00159h

28. Scukins A, Nerukh D, Pavlov E, Karabasov S, Markesteijn A. Multiscale molecular dynamics/hydrodynamics implementation of two dimensional "Mercedes Benz" water model. Eur Phys J Spec Top. 2015;224(12):2217-2238. doi:10.1140/epjst/e2015-02409-8 
29. Lighthill MJ. On Sound Generated Aerodynamically. I. General Theory. Proc R Soc A Math Phys Eng Sci. 1952;211(1107):564-587. doi:10.1098/rspa.1952.0060

30. Landau LD, Lifshitz EM. Statistical Physics, Part 1. Amsterdam: Elsevier; 1980.

31. Korotkin I, Karabasov S, Nerukh D, et al. A hybrid molecular dynamics/fluctuating hydrodynamics method for modelling liquids at multiple scales in space and time. J Chem Phys. 2015;143(1). doi:10.1063/1.4923011

32. Korotkin I, Nerukh D, Tarasova E, Farafonov V, Karabasov S. Two-phase flow analogy as an effective boundary condition for modelling liquids at atomistic resolution. J Comput Sci. 2016;17. doi:10.1016/j.jocs.2016.03.012

33. Tarasova E, Korotkin I, Farafonov V, Karabasov S, Nerukh D. Complete virus capsid at allatom resolution: Simulations using molecular dynamics and hybrid molecular dynamics/hydrodynamics methods reveal semipermeable membrane function. J Mol Liq. 2017;245. doi:10.1016/j.molliq.2017.06.124

34. Abraham MJ, Murtola T, Schulz R, et al. GROMACS: High performance molecular simulations through multi-level parallelism from laptops to supercomputers. SoftwareX. 2015;1-2:19-25. doi:10.1016/j.softx.2015.06.001

35. Van Der Spoel D, Lindahl E, Hess B, Groenhof G, Mark AE, Berendsen HJC. GROMACS: Fast, flexible, and free. J Comput Chem. 2005;26(16):1701-1718. doi:10.1002/jcc.20291

36. Hu J, Korotkin IA, Karabasov SA. A multi-resolution particle/fluctuating hydrodynamics model for hybrid simulations of liquids based on the two-phase flow analogy. J Chem Phys. 2018;149(8):084108. doi:10.1063/1.5040962

37. Markesteijn A, Karabasov S, Glotov V, Goloviznin V. A new non-linear two-time-level Central Leapfrog scheme in staggered conservation-flux variables for fluctuating hydrodynamics equations with GPU implementation. Comput Methods Appl Mech Eng. 2014;281:29-53. 
doi:10.1016/j.cma.2014.07.027

38. De Fabritiis G, Serrano M, Delgado-Buscalioni R, Coveney P V. Fluctuating hydrodynamic modeling of fluids at the nanoscale. Phys Rev E. 2007;75(2):026307. doi:10.1103/PhysRevE.75.026307

39. Buckley SE, Leverett MC. Mechanism of Fluid Displacement in Sands. Trans AIME. 1942;146(01):107-116. doi:10.2118/942107-G

40. Kotsalis EM, Walther JH, Kaxiras E, Koumoutsakos P. Control algorithm for multiscale flow simulations of water. Phys Rev E. 2009;79(4):045701. doi:10.1103/PhysRevE.79.045701

41. Delgado-Buscalioni R, Coveney P V. USHER: An algorithm for particle insertion in dense fluids. J Chem Phys. 2003;119(2):978-987. doi:10.1063/1.1579475

42. Berendsen HJC, van der Spoel D, van Drunen R. GROMACS: A message-passing parallel molecular dynamics implementation. Comput Phys Commun. 1995;91(1-3):43-56. doi:10.1016/0010-4655(95)00042-E

43. Páll S, Hess B. A flexible algorithm for calculating pair interactions on SIMD architectures. Comput Phys Commun. 2013;184(12):2641-2650. doi:10.1016/j.cpc.2013.06.003

44. Kutzner C, Páll S, Fechner M, Esztermann A, de Groot BL, Grubmüller H. Best bang for your buck: GPU nodes for GROMACS biomolecular simulations. $J$ Comput Chem. 2015;36(26):1990-2008. doi:10.1002/jcc.24030

45. Kenis PJ, Ismagilov RF, Whitesides GM. Microfabrication Inside Capillaries Using Multiphase Laminar Flow Patterning. Science (80- ). 1999;285(5424):83-85. doi:10.1126/science. 285.5424 .83

46. Kenis PJA, Ismagilov RF, Takayama S, Whitesides GM, Li S, White HS. Fabrication inside Microchannels Using Fluid Flow. Acc Chem Res. 2000;33(12):841-847. doi:10.1021/ar000062u 
47. Wong PK, Lee Y-K, Ho C-M. Deformation of DNA molecules by hydrodynamic focusing. $J$ Fluid Mech. 2003;497:S002211200300658X. doi:10.1017/S002211200300658X

48. Evans DJ, Morriss G. Statistical Mechanics of Nonequilibrium Liquids, Second Edition.; 2008. doi:10.1017/CBO9780511535307

49. Todd BD. Computer simulation of simple and complex atomistic fluids by nonequilibrium molecular dynamics techniques. In: Computer Physics Communications. Vol 142. ; 2001:1421. doi:10.1016/S0010-4655(01)00304-6

50. Hoover WG, Hoover CG. Links between microscopic and macroscopic fluid mechanics. Mol Phys. 2003;101(11):1559-1573. doi:10.1080/0026897021000026647

51. De Fabritiis G, Delgado-Buscalioni R, Coveney P V. Multiscale modeling of liquids with molecular specificity. Phys Rev Lett. 2006;97(13):1-4. doi:10.1103/PhysRevLett.97.134501

52. Delgado-Buscalioni R, Kremer K, Praprotnik M. Coupling atomistic and continuum hydrodynamics through a mesoscopic model: Application to liquid water. J Chem Phys. 2009;131(24):1-8. doi:10.1063/1.3272265

53. Delgado-Buscalioni R, De Fabritiis G. Embedding molecular dynamics within fluctuating hydrodynamics in multiscale simulations of liquids. Phys Rev E - Stat Nonlinear, Soft Matter Phys. 2007;76(3):1-13. doi:10.1103/PhysRevE.76.036709 